\title{
Estimating exponential scheduling preferences
}

Hjorth, Katrine; Börjesson, Maria; Engelson, Leonid; Fosgerau, Mogens

Published in:

Transportation Research. Part B: Methodological

Link to article, DOI:

10.1016/j.trb.2015.03.014

Publication date:

2015

Document Version

Peer reviewed version

Link back to DTU Orbit

Citation (APA):

Hjorth, K., Börjesson, M., Engelson, L., \& Fosgerau, M. (2015). Estimating exponential scheduling preferences. Transportation Research. Part B: Methodological, 81, 230-251. https://doi.org/10.1016/j.trb.2015.03.014

\section{General rights}

Copyright and moral rights for the publications made accessible in the public portal are retained by the authors and/or other copyright owners and it is a condition of accessing publications that users recognise and abide by the legal requirements associated with these rights.

- Users may download and print one copy of any publication from the public portal for the purpose of private study or research.

- You may not further distribute the material or use it for any profit-making activity or commercial gain

- You may freely distribute the URL identifying the publication in the public portal

If you believe that this document breaches copyright please contact us providing details, and we will remove access to the work immediately and investigate your claim. 


\title{
Estimating exponential scheduling preferences
}

\author{
Katrine Hjorth, Maria Börjesson`, Leonid Engelson $¥$ Mogens Fosgerau ${ }^{\S}$
}

Friday $20^{\text {th }}$ March, 2015

\begin{abstract}
Different assumptions about travelers' scheduling preferences yield different measures of the cost of travel time variability. Only few forms of scheduling preferences provide non-trivial measures which are additive over links in transport networks where link travel times are arbitrarily distributed independent random variables: Assuming smooth preferences, this holds only for specifications with a constant marginal utility of time at the origin and an exponential or affine marginal utility of time at the destination. We apply a generalized version of this model to stated preference data of car drivers' route and mode choice under uncertain travel times. Our analysis exposes some important methodological issues related to complex non-linear scheduling models: One issue is identifying the point in time where the marginal utility of being at the destination becomes larger than the
\end{abstract}

*Technical University of Denmark, Denmark, khj@transport.dtu.dk

${ }^{\dagger}$ Royal Institute of Technology, Sweden

†Royal Institute of Technology, Sweden

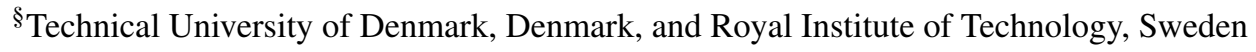


marginal utility of being at the origin. Another issue is that models with the exponential marginal utility formulation suffer from empirical identification problems. Though our results are not decisive, they partly support the constant-affine specification, in which the value of travel time variability is proportional to the variance of travel time.

Keywords: Travel time variability, scheduling preferences

\section{Introduction}

Measurement of travelers' trip scheduling preferences is important because they drive departure time choices (Vickrey, 1973) and underlie travelers' values of travel time and travel time variability (Noland and Small, 1995; Bates et al., 2001; Fosgerau and Karlström, 2010). The purpose of this paper is to empirically estimate scheduling preference specifications with a certain convenient property: That the cost of travel time variability is additive over independent traffic links. Our results lend some support to the simplest scheduling preference specification having this property, defined by a constant marginal utility of time at the origin and an affine marginal utility of time at the destination. This specification implies that the value of travel time variability is proportional to the variance of travel time.

A large class of scheduling preferences can be formally represented as time-dependent rates of utility derived at different locations. Vickrey (1973) formulated the departure time choice problem in terms of minimizing the sum of two integrals with integrands representing the marginal utility of time (MUT) at origin $(H)$ and at destination $(W)$ relative to the time spent traveling with both MUT varying by time of day. This formulation was further developed by Tseng and Verhoef (2008) and generalized to a two-trip chain by Jenelius et al. (2011). 
The popular 'constant $H$ - step $W$ ' scheduling formulation (hereafter referred to as const-step) proposed by Vickrey (1969) and Small (1982) is a special case of the model suggested by Vickrey (1973), where $H$ is constant and $W$ is a step function with two fixed utility rates for time spent at the destination before and after the Preferred Arrival Time (PAT) ${ }^{1}$ Noland and Small (1995) used expected utility theory to derive a reducedform utility function based on const-step scheduling preferences, under the assumption that the travel time is exponentially or uniformly distributed and that travellers choose their departure time optimally. This reduced-form model included the cost of travel time variability (TTV), expressed by the standard deviation of travel time. Fosgerau and Karlström (2010) generalized this result to a general travel time distribution: They showed that, in a reduced-form model based on const-step scheduling preferences, the standard deviation of travel time is an appropriate measure for valuation of TTV, with a value depending on the shape of the travel time distribution. Fosgerau and Engelson (2011) showed that a scheduling formulation with affine $H$ and $W$ implies that the measure of TTV is proportional to the variance of travel time, with a coefficient independent of the distribution shape. Engelson (2011) derived explicit measures of the valuation of TTV when both functions $H$ and $W$ are second-order polynomials or exponential functions. These are probably the most general functions for $H$ and $W$ with a closed form expression for the measure of TTV.

The above-mentioned results concern car travel, where departure time choice is continuous. However, the framework has also been generalized to the case of a scheduled transit service: Both Fosgerau and Engelson (2011) and Engelson and Fosgerau (2011) (the latter considering scheduling formulations with constant $H$ and affine or exponential $W$ ) extended their results to the scheduled service case considering costs

\footnotetext{
${ }^{1}$ But actually, Vickrey (1969)'s and Small (1982)'s models were not formulated in terms of utility rates, but in terms of corresponding cost functions.
} 
of travel time and headway in settings with uncertain travel times and fixed headways. The scheduling framework has also been applied in analyses considering settings with known travel times (no TTV) and known or random headways: For known headways, Fosgerau (2009) derived the value of headway for general scheduling preferences and the case of const-step preferences. For random headways, Benezech and Coulombel (2013) derived values of (mean) headway and service reliability (standard deviation of headway) in the case of const-step preferences.

Travel time reliability is becoming increasingly important in transport modelling and assignment (Lam et al., 2014). In transport network applications, TTV and other components of the generalized travel costs are usually measured on links. In order to convert generalized travel cost from link to trip level, network assignment algorithms generally assume that travel costs are additive across links within each path. Hence, it is desirable that the measure of travel time variability is additive. Using the standard deviation of travel time as a measure of TTV does not satisfy this, as the standard deviation is additive only in the unrealistic case of perfect positive correlation between the link travel times. The variance, however, is additive if the link travel times are independent. The assumption of independence is strong, but results from Fosgerau and Fukuda (2012) and Eliasson (2007) suggest that the error made when converting the TTV from link to trip level while assuming independence of the travel time distributions across links is small in many cases.

Engelson and Fosgerau (2011) showed that the only form of smooth scheduling preferences providing an (non-trivial) additive measure of TTV when link travel times are arbitrarily distributed independent random variables, is a constant $H$ and an exponential or affine $W 2^{2}$. These specifications thus give a convenient expression of travel cost for

\footnotetext{
${ }^{2}$ The 'constant $H$ - exponential $W$ ' formulation implies that the cost of TTV is proportional to the cumulant generating function $\ln E \exp (\beta T)$ where $T$ is the random travel time and $E$ is the expectation.
} 
calculation of the value of TTV in transport networks assuming that the distribution of travel times for different links are independent.

Even if scheduling models implying additive measures of TTV are desirable for application purposes, the most appropriate scheduling specification and corresponding measure is an empirical matter. No previous study has estimated the 'constant $H$ - exponential $W^{\prime}$ (const-exp) scheduling model and tested its empirical properties. Most departure time choice modeling studies have applied the const-step formulation by Small (1982) and Abkowitz (1981), see the overview by de Jong et al. (2003). HendrickSon and Plank (1984) estimated quadratic penalty functions for early and late arrivals that correspond to a $W$ function defined by an affine expression before PAT and another affine expression after PAT. Ettema et al. (2004) estimated parameters of the time varying utility rates based on departure time choice of complete tours while Tseng and Verhoef (2008) applied non-parametric techniques to estimate such rates for trips from home to work. There is also a vast body of empirical studies estimating the disutility of travel time variability directly, using measures of travel time variability such as standard deviation, variance or mean-delay, using SP data (see, e.g., the review in Carrion and Levinson, 2012). Estimating both a scheduling and reduced-form models, Börjesson et al. (2012) find that scheduling preferences do not capture the traveler's entire disutility of delays.

Very few scheduling models have been estimated on real data. The main contribution of this paper is showing that it is possible to empirically estimate scheduling functions that have the desirable additivity property, the const-exp specification, and its limiting case, the const-affine specification. Based on stated preference (SP) survey data we are also able to estimate the more general, exponential-exponential specification of $H$ and $W$ As mentioned above, the 'constant $H$ - affine $W$ ' formulation implies that the cost of TTV is proportional to the variance of travel time. 
(exp-exp), suggested by Engelson(2011), and the conventional const-step specification. We attempt to evaluate whether the specifications providing additive measures for the travel time variability can be preferred to these benchmark models. We find that the parameters of the exponential scheduling functions $H$ or $W$ are poorly identified, with very high standard errors if the models can be estimated at all. However, applying multiplicative error terms facilitates the identification in many cases. It may be possible to design a SP experiment that allows identification of exponential scheduling functions with higher precision, but the appropriate scheduling function is usually not known when the SP experiments are designed. A second contribution of this paper is to reveal these methodological problems.

Although we do not account for this in our models because it would further complicate the estimation, there is probably a substantial heterogeneity in scheduling preferences and constraints across travelers and trips. This is probably an additional reason for the identification problems, since even the appropriate scheduling functional forms may differ across trips and travelers.

Though our results for the exponential specifications are not decisive, we can compare the const-affine and the conventional const-step specifications which both yield plausible results. These results lend some support to the const-affine specification, in which the value of travel time variability is proportional to the variance of travel time. The const-step specification performs well for travelers with fixed restrictions on their arrival time, which is to be expected since this model assumes a jump in scheduling costs when arriving even shortly after PAT compared to arriving shortly before.

The paper is organized as follows. In the next section we give an analytic formulation of the $H$ and $W$ functions and the expected costs in the combined mode and departure time choice model. Section 3 describes data used for model estimation. Section 


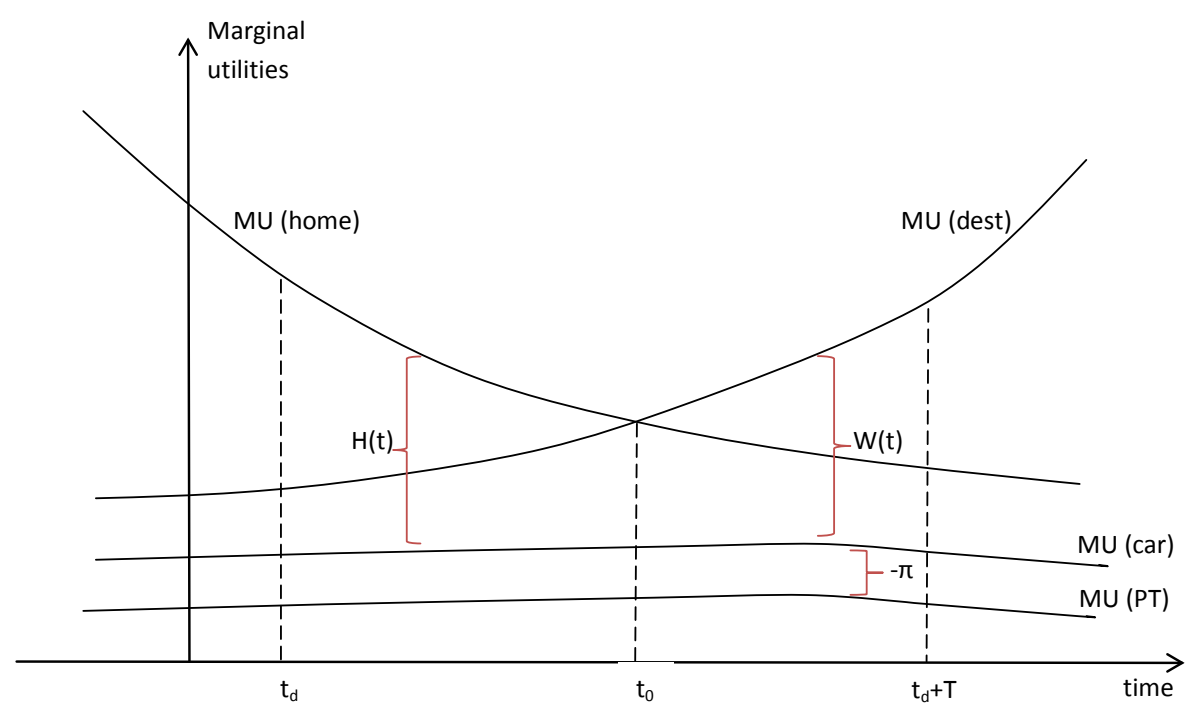

Figure 1: Marginal utilities of time spent at home and at the estimation, time spent traveling with car, and time spent traveling with public transport.

4 explains how the model has been adjusted for the estimation using the available data while section 5 demonstrates and discusses the obtained results. Section 6 concludes.

\section{The Model}

\subsection{General setup}

Consider travelers having a specific origin and destination, between which they have the option to travel by car or by public transport during some part of the day. We assume that the travelers are equipped with scheduling preferences and choose mode and departure time such that they maximize their expected utility. We set up a scheduling model based on the assumption that the total utility is affected by lost time at the origin, lost time at the destination, time spent traveling and monetary costs.

Denote the marginal utility of being at the origin relative to the marginal utility of 
time spent traveling by car at time $t$ as $H(t)$, and the marginal utility of being at the destination relative to the marginal utility of time spent traveling by car at time $t$ as $W(t) . \pi$ is the marginal utility of traveling by public transport relative to the marginal utility of traveling by car, which is assumed to be constant over time $t$ (a positive value of $\pi$ corresponds to a higher marginal utility of travelling by car than by PT). Assume that $\mathrm{H}$ is non-increasing and $\mathrm{W}$ is non-decreasing and that there is a time $t_{0}$ such that $H(t)>W(t)$ for $t<t_{0}$ and $H(t)<W(t)$ for $t>t_{0}$. Then the individual prefers to be at the origin before $t_{0}$ and at the destination after $t_{0}$. This idea is expressed graphically in Figure 1.

For departure time $t_{d}$, car travel time $T$, monetary car travel cost $C$, public transport travel time $T_{P T}$, and monetary public transport cost $C_{P T}$, the generalized costs of traveling with car and public transport, respectively, are assumed to be $\mathrm{b}^{3}$

$$
\begin{aligned}
G_{\mathrm{car}}\left(t_{d}, T, C\right) & =C+\int_{t_{d}}^{t_{0}} H(t) d t+\int_{t_{0}}^{t_{d}+T} W(t) d t \\
G_{P T}\left(t_{d}, T_{P T}, C_{P T}\right) & =C_{P T}+\int_{t_{d}}^{t_{0}} H(t) d t+\int_{t_{0}}^{t_{d}+T_{P T}} W(t) d t+\pi T_{P T}+K .
\end{aligned}
$$

Here, $K$ is a mode-specific cost for public transport. $K$ represents all those costs of traveling by PT rather than car that are not related to the departure time or the duration of the journey.

We consider four formulations of the scheduling preferences. In the first and most general formulation (exp-exp), $H(t)$ and $W(t)$ are both exponential functions, as suggested in Engelson (2011). In the second formulation (const-exp), $H$ is constant and $W$ exponential, as in Engelson and Fosgerau (2011). The third formulation (const-affine) has constant $H$ and affine $W$, which is a special case of the formulation in Fosgerau and Engelson (2011). We note that the second formulation is a special case of the first, while the third formulation is a limiting case of the second. Finally we apply the well-known

\footnotetext{
${ }^{3}$ We normalize the marginal generalized cost of monetary cost to one.
} 
const-step specification of Vickrey (1969) and Small (1982), where $H$ is constant and $W$ is a step function.

\subsection{Exp-exp specification}

We begin by deriving our most general model formulation, where $H$ and $W$ are exponential functions. The model is constrained by restricting the exponent to be the same in $H$ and $W$ :

$$
\begin{gathered}
H(t)=H_{0}+n e^{\eta\left(t-t_{0}\right)}, \\
W(t)=W_{0}+m e^{\eta\left(t-t_{0}\right)},
\end{gathered}
$$

where $\eta \neq 0$.

This parameterization of $H$ and $W$ in terms of $t-t_{0}$ corresponds to assuming that preferences are homogeneous up to a horizontal transformation, i.e. the marginal utility of being at the origin/destination, relative to the marginal utility of traveling by car, at a time $x$ minutes past $t_{0}$ is the same for all individuals, even though $t_{0}$ may differ between individuals.

In order to have $H(t)>W(t)$ for $t<t_{0}$ and $H(t)<W(t)$ for $t>t_{0}$, which with continuous functions $H$ and $W$ implies $H\left(t_{0}\right)=W\left(t_{0}\right)$, we must have that $H_{0}=W_{0}+$ $m-n$. The marginal utility functions are thus

$$
\begin{aligned}
& H(t)=W_{0}+m-n+n e^{\eta\left(t-t_{0}\right)}, \\
& W(t)=W_{0}+m e^{\eta\left(t-t_{0}\right)} .
\end{aligned}
$$

Furthermore, for $H$ to be non-increasing and for $W$ to be non-decreasing, we must have that

$$
\eta \neq 0, \quad m \eta \geq 0, \quad n \eta \leq 0
$$


Using (1), (2), (5) and (6), the generalized costs of traveling by car and public transport, respectively, become

$$
\begin{aligned}
G_{\mathrm{car}}\left(t_{d}, T, C\right)= & C+\frac{n-m}{\eta}+W_{0} T+(n-m)\left(t_{d}-t_{0}\right) \\
& +\frac{1}{\eta} e^{\eta\left(t_{d}-t_{0}\right)}\left(m e^{\eta T}-n\right), \\
G_{P T}\left(t_{d, P T}, T_{P T}, C_{P T}\right)= & C_{P T}+K+\frac{n-m}{\eta}+\left(W_{0}+\pi\right) T_{P T}+(n-m)\left(t_{d, P T}-t_{0}\right) \\
& +\frac{1}{\eta} e^{\eta\left(t_{d, P T}-t_{0}\right)}\left(m e^{\eta T_{P T}}-n\right) .
\end{aligned}
$$

\subsection{Specifications with additivity property (const-exp and const-affine)}

The const-exp preference specification, where $H$ is constant and $W$ exponential, is a special case of the model defined by (7) and (8) where $n=0$. The const-affine preference specification, where $H$ is constant and $W$ affine, is given by

$$
\begin{aligned}
H(t) & =\zeta \\
W(t) & =\zeta+\omega \cdot\left(t-t_{0}\right)
\end{aligned}
$$

Note that this is a limiting case of the const-exp model formulation.$^{4}$ The const-affine specification is also a special case of the model suggested by Fosgerau and Engelson (2011) with a scheduling preference specification using affine functions for both MUT at origin and MUT at destination. Using (1), (2), (9) and (10), we find that the generalized costs in the third formulation are

$$
\begin{aligned}
G_{\mathrm{car}}\left(t_{d}, T, C\right) & =C+\zeta T+\frac{\omega}{2}\left(t_{d}-t_{0}+T\right)^{2} \\
G_{P T}\left(t_{d, P T}, T_{P T}, C_{P T}\right) & =C_{P T}+K+(\pi+\zeta) T_{P T}+\frac{\omega}{2}\left(t_{d, P T}-t_{0}+T_{P T}\right)^{2} .
\end{aligned}
$$

${ }^{4}$ The const-affine formulation is obtained from eqs (5) and (6) by first setting $n=0$ and then defining $\zeta=W_{0}+m$ and $\omega=m \eta$. This yields the reparameterized utility rates $H(t)=\zeta$ and $W(t)=$ $\zeta+\frac{\omega}{\eta}\left(e^{\eta\left(t-t_{0}\right)}-1\right)$. Letting $\eta$ tend to zero gives eqs (9) and (10). 


\subsection{Const-step specification}

In the standard const-step formulation, we have

$$
\begin{aligned}
& H(t)=\alpha, \\
& W(t)= \begin{cases}\alpha-\beta & \text { if } t \leq t_{0}, \\
\alpha+\gamma & \text { if } t>t_{0},\end{cases}
\end{aligned}
$$

where $\alpha, \beta$, and $\gamma$ are non-negative parameters. Using (1), (2), (13) and (14) the generalizeds cost of traveling with car and public transport become

$$
\begin{aligned}
G_{\mathrm{car}}\left(t_{d}, T, C\right) & =C+\alpha T+\beta S D E\left(t_{d}, T\right)+\gamma S D L\left(t_{d}, T\right), \\
G_{P T}\left(t_{d, P T}, T_{P T}, C_{P T}\right) & =C_{P T}+K+(\pi+\alpha) T_{P T}+\beta S D E\left(t_{d, P T}, T_{P T}\right)+\gamma S D L\left(t_{d, P T}, T_{P T}\right),
\end{aligned}
$$

where SDE and SDL are 'schedule delay early' and 'schedule delay late', respectively, and given by

$$
\begin{aligned}
& S D E\left(t_{d}, T\right)=\max \left(t_{0}-t_{d}-T, 0\right), \\
& S D L\left(t_{d}, T\right)=\max \left(t_{d}+T-t_{0}, 0\right) .
\end{aligned}
$$




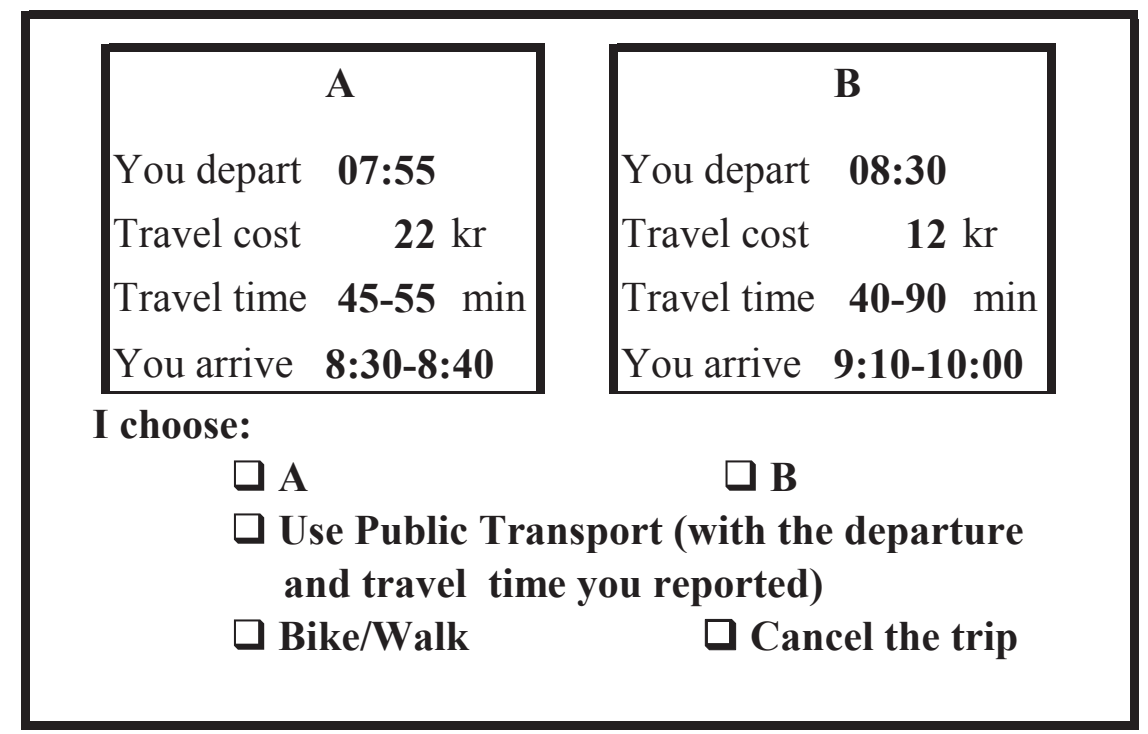

Figure 2: Example of SP choice with explicit car costs

\section{Data}

We use data from a SP study among car drivers in Stockholm from Börjesson (2008. 2009). Respondents were sampled from the group of car drivers crossing Tranebergsbron (a bridge connecting central Stockholm to its western suburbs) on weekday mornings in April and May 2005 (before the introduction of congestion charges). The principal recruitment method was to randomly record license plate numbers of passing cars. The registration numbers were then matched with names, addresses and phone numbers of the car owners. The car owners were contacted by telephone later the same day or the following day. The telephone interview contained questions about the observed trip, including purpose, departure time and travel time, and background questions about socioeconomic status. The respondents were asked whether they had fixed or flexible working time. A fixed working time means that the respondent has to start work at a particular time, while a flexible working time means that he can choose when to start: 
Either within some time interval or completely freely. Travelers with flexible working time were also asked if there was any particular time when they needed to be at the destination the day they were observed. When we distinguish between fixed and flexible working time in our analysis, we refer to the respondents' usual work conditions and ignore whether there was a specific appointment on the day of interest. $5^{5}$

During the interview the car drivers were requested to participate in a SP experiment. Respondents who agreed to participate in the SP experiment were sent a customized paper questionnaire the following day, with additional questions regarding the observed trip (which we refer to as the revealed preference or RP trip) and socioeconomic background, and eight SP choices. Each SP choice had five alternatives:

- two car alternatives differing with respect to departure time, travel time distribution and monetary travel costs,

- a public transport (PT) alternative with a fixed travel time (respondents were instructed to assume their stated public transport time in all choices),

- a walk/cycle alternative,

- cancel the trip.

The travel time distribution in the car alternatives was presented as an interval in which 'travel time normally varies'. Participants were instructed that this meant that travel time could fall outside the interval, and that this would happen approximately once a month if the trip was made every weekday. To prepare the respondents to think about variability, those reporting that they were making similar trips on a regular basis (almost 90 percent of our estimation sample) were asked to report the minimum and

\footnotetext{
${ }^{5} \mathrm{We}$ also analyzed models where we took this information into account and distinguished between fixed and flexible arrival time on the specific day of interest, but this gave a worse model fit.
} 
maximum travel time that they usually experienced. Their answers indicate that they understood and were able to interpret the interval, as 90 percent reported an interval with a midpoint less than 10 minutes from the reported travel time for the observed trip. The monetary travel cost was only included as an experimental factor in the last four choices. In this paper, we use only the data from these choices, exemplified in Figure 2.

An orthogonal design based on the difference between the two car alternatives was used. In one of the two car alternatives in each choice, the departure time was shifted 5-15 minutes later or earlier from their stated departure time. The departure time in the second alternative was shifted further away in the same direction (later or earlier), and the size of the shift was determined by the design.

The travel time intervals in the car alternatives were determined by two variables, midpoint and width. The difference of the interval midpoints between the two alternatives took four levels, ranging from 10 to 25 minutes. The interval width was then derived as a percentage of the midpoint, with the four levels ranging from 0 to $80 \%$. Cost differences between the two car alternatives were assigned four levels in the range of SEK 10 to 40 (approximately EUR 1 to 4). The range of the design variables, and some variables related to the trips, for the final estimation sample (see below) are shown in Table1,

For a given individual, the travel time of the public transport alternative was fixed in all choices and equal to a value stated by the participant in the questionnaire before answering the SP choices. When missing, the public transport travel time was calculated based on the frequencies and itineraries of the public transport lines using the software Emme given the origin and destination addresses of the respondents.

The departure time in the public transport alternative was not explicitly mentioned. Moreover, the best public transport alternative for most of the recruited travelers would 
have been the Metro. During the morning the service frequency of the Metro is 3-5 minutes, and hence it is reasonable to assume that the respondents choose the departure time freely when choosing public transport. Participants were told that the monetary cost of the public transport alternative was the current ticket price for the trip, unless they held a travel card for the Stockholm public transport system, in which case they were instructed to consider the public transport alternative as having a zero monetary travel cost. For respondents without a travel card, the monetary travel cost is unknown. It is, however, strongly correlated with travel distance and travel time.

We restrict our analysis to trips from home to work, corresponding to 404 (of 581) participants with a total of 1600 choices. We do this for two reasons. First, it is more likely to suit our model's assumption of homogeneous preferences. Second, the average price per person and trip depends on the size of the company, which is unknown. Focusing on work trips mitigates this problem, since commuting trip usually are made without company.

Since the alternatives 'Bike/Walk' and 'Cancel the trip' were chosen in only 3.3 $\%$ of the choices, we restrict our analysis to consider the car and the public transport alternatives and so end up with a sample of 403 individuals and 1547 observations.

\section{Model Estimation}

This section describes how we use logit models to estimate the model parameters. For simplicity, we assume preference homogeneity in the parameters $\eta, W_{0}, n, m, \zeta, \omega, \alpha$, $\beta, \gamma, \lambda, \pi$ and $K$. However, we assume that $t_{0}$ may vary between individuals to reflect differences in trip timing preferences. 
Table 1: Means and quantiles of variables. Time variables are in minutes, cost variables in SEK.

\begin{tabular}{|c|c|c|c|c|c|c|}
\hline & Mean & Min & $25 \%$ Quant & Median & $75 \%$ Quant & Max \\
\hline \multicolumn{7}{|l|}{ SP attributes } \\
\hline Midpoint of travel time interval, alt A & 38.3 & 10.0 & 27.5 & 35.0 & 50.0 & 135 \\
\hline Midpoint of travel time interval, alt B & 40.7 & 7.50 & 25.0 & 40.0 & 52.5 & 145 \\
\hline Width of travel time interval, alt A & 15.5 & 0.00 & 0.00 & 10.0 & 25.0 & 80.0 \\
\hline Width of travel time interval, alt B & 16.6 & 0.00 & 5.00 & 10.0 & 25.0 & 110 \\
\hline Departure time minus actual departure time, alt A & 0.02 & -75.0 & -18.0 & -4.00 & 15.0 & 62.0 \\
\hline Departure time minus actual departure time, alt B & -0.07 & -75.0 & -20.0 & -5.00 & 15.0 & 62.0 \\
\hline Midpoint of arrival time interval minus actual arrival time, alt $\mathrm{A}$ & 2.47 & -65.0 & -8.75 & 5.00 & 15.0 & 70.0 \\
\hline Midpoint of arrival time interval minus actual arrival time, alt B & 4.84 & -85.0 & -20.0 & -7.00 & 35.0 & 85.0 \\
\hline Monetary cost, alt A & 25.2 & 5.00 & 15.0 & 20.0 & 25.0 & 60.0 \\
\hline Monetary cost, alt B & 25.7 & 5.00 & 12.0 & 22.0 & 45.0 & 60.0 \\
\hline \multicolumn{7}{|l|}{ Choice variable } \\
\hline Proportion where choice $=$ alt $\mathrm{A}$ & 0.539 & & & & & \\
\hline Proportion where choice $=$ alt $\mathrm{B}$ & 0.348 & & & & & \\
\hline Proportion where choice $=\mathrm{PT}$ & 0.112 & & & & & \\
\hline \multicolumn{7}{|l|}{ Background info } \\
\hline Actual travel time & 35.8 & 10.0 & 25.0 & 35.0 & 45.0 & 120 \\
\hline Width of usual travel time interval & 20.9 & 0.00 & 10.0 & 17.0 & 30.0 & 90.0 \\
\hline PT travel time & 67.6 & 8.30 & 50.0 & 60.0 & 80.0 & 180 \\
\hline Proportion of frequent trips (at least a couple of times a week) & 0.862 & & & & & \\
\hline Proportion of respondents with fixed working hours & 0.341 & & & & & \\
\hline Proportion of respondents without travel card & 0.861 & & & & & \\
\hline
\end{tabular}




\subsection{Choice models}

We assume that respondents choose between the car and public transport alternatives in the SP choices by assigning a generalized $\operatorname{cost} \mathscr{G}_{i}$ to each alternative and choosing the alternative with the smaller cost, while allowing for a random error. To model this, we define a logit model with three alternatives $i \in\{\operatorname{car} 1, \operatorname{car} 2, P T\}$ : Two car alternatives and one public transport alternative.

We consider two choice models: The first is a standard logit specification where the probability of choosing alternative $i$ is given by:

$$
P_{i}=\operatorname{Prob}\left(\mathscr{G}_{i}+\frac{1}{\mu} \varepsilon_{i}<\mathscr{G}_{j}+\frac{1}{\mu} \varepsilon_{j}, \forall j \neq i\right),
$$

where the $\varepsilon$ 's are independent and identically extreme value distributed, and $\mu$ is a positive error scale parameter. In this model the error terms are additive to the alternative costs.

We also consider a model with multiplicative errors (cf. Fosgerau and Bierlaire, 2009) specified as:

$$
P_{i}=\operatorname{Prob}\left(\ln \left(\mathscr{G}_{i}\right)+\frac{1}{\mu} \varepsilon_{i}<\ln \left(\mathscr{G}_{j}\right)+\frac{1}{\mu} \varepsilon_{j}, \forall j \neq i\right) .
$$

All four preference specifications are estimated using both choice model specification (19) and (20). For simplicity, we disregard the panel structure of the data and use pooled cross-sectional models, i.e. multinomial logit models. ${ }^{6}$ This yields consistent but not efficient estimates, and requires that we use a robust 'sandwich' estimator of the

\footnotetext{
${ }^{6} \mathrm{We}$ did attempt to estimate mixed logit models where the PT-specific constant $K$ has a random distribution in the population, but is fixed within choices of the same individual. We tried both a Normal and a Uniform distribution for $K$, in combination with different starting values and different optimization algorithms. We were able to achieve convergence only for the const-affine model, and this did not converge to an optimal solution (the covariance matrix could not be computed).
} 
covariance matrix to compute the standard errors. Another implication is that the usual Likelihood Ratio test is not valid (see e.g. Wooldridge, 2002, sec. 13.8).

\subsection{Specification of generalized costs}

In this section we specify the generalized costs $\mathscr{G}_{i}$ that the respondents assign to each alternative. For the car alternatives, we assume that $\mathscr{G}_{i}$ is the expected generalized cost $E G_{\text {car }}\left(t_{d}, C\right)$ from the model in section 2. The expected generalized cost is evaluated for the departure time and monetary cost given as alternative attributes in the questionnaire. Note that this departure time is not necessarily optimal. Table 2 gives the expressions for $\mathscr{G}_{i}$ for the car alternatives in the four different preference specifications (see the derivation in Appendix A.

Table 2 also shows the corresponding expressions for the public transport alternative, which are derived in a somewhat different manner, as this alternative differs from the car alternatives in two important ways. First, for the public transport alternative the monetary travel cost depends on whether the respondent holds a travel card. If so, the monetary cost is zero. Otherwise, it equals the current ticket price. We do not know this ticket price but we know that it is correlated with travel distance and travel time. For this reason we approximate the ticket price for travelers without a travel card by $C_{P T}=\lambda T_{P T}$, where $\lambda$ is a parameter that will be estimated. Second, we assume that the public transport travel time is deterministic (known with certainty), and respondents can choose departure time freely (see section 3). We therefore assume that the chosen departure time is optimal. The derivation of $\mathscr{G}_{P T}$ is shown in Appendix A.

As can be seen from Table $2, \mathscr{G}_{i}$ for the car alternatives depends on $t_{0}$, which is unobserved. We use the respondent's stated departure time $\left(t_{d, R P}\right)$ and the midpoint of the usual travel time interval stated by the respondent $\left(\bar{T}_{R P}\right)$ to derive an individual- 
Table 2: The empirical generalized costs for different preference specifications

\begin{tabular}{|c|c|c|}
\hline $\begin{array}{l}\text { Preference } \\
\text { specification }\end{array}$ & $\begin{array}{l}\text { Costs } \mathscr{G}_{c a r 1} \text { and } \mathscr{G}_{c a r 2} \text { assigned to car alternatives, } \\
\operatorname{cost} \mathscr{G}_{P T} \text { assigned to PT alternative and } t_{0}^{\dagger}\end{array}$ & \\
\hline exp-exp & $\begin{array}{l}\mathscr{G}_{i}=C_{i}+\frac{n-m}{\eta}+W_{0} E T_{i}+(n-m)\left(t_{d, i}-t_{0}\right)+\frac{1}{\eta} e^{\eta\left(t_{d, i}-t_{0}\right)}\left(m E e^{\eta T_{i}}-n\right) \\
\mathscr{G}_{P T}=\lambda T_{P T} 1_{\mathrm{PAY}}+K+\left(W_{0}+\pi\right) T_{P T}+\frac{m-n}{\eta}\left(\ln \left(m e^{\eta T_{P T}}-n\right)-\ln (m-n)\right) \\
t_{0}=t_{d, R P}+\frac{1}{\eta}\left(\ln \left(m e^{\eta \bar{T}_{R P}}-n\right)-\ln (m-n)\right)\end{array}$ & $i \in\{\operatorname{car} 1, \operatorname{car} 2\}$ \\
\hline const-exp & $\begin{array}{l}\mathscr{G}_{i}=C_{i}+\frac{-m}{\eta}+W_{0} E T_{i}-m\left(t_{d, i}-t_{0}\right)+\frac{1}{\eta} e^{\eta\left(t_{d, i}-t_{0}\right)}\left(m E e^{\eta T_{i}}\right), \\
\mathscr{G}_{P T}=\lambda T_{P T} 1_{\mathrm{PAY}}+K+\left(W_{0}+\pi+m\right) T_{P T} \\
t_{0}=t_{d, R P}+\bar{T}_{R P}\end{array}$ & $i \in\{\operatorname{car} 1, \operatorname{car} 2\}$ \\
\hline const-affine & $\begin{array}{l}\mathscr{G}_{i}=C_{i}+\frac{\omega}{2}\left(t_{d, i}-t_{0}\right)^{2}+\left(\zeta+\omega \cdot\left(t_{d, i}-t_{0}\right)\right) E T_{i}+\frac{\omega}{2} E T_{i}^{2}, \\
\mathscr{G}_{P T}=\lambda T_{P T} 1_{\mathrm{PAY}}+K+(\zeta+\pi) T_{P T} \\
t_{0}=t_{d, R P}+\bar{T}_{R P}\end{array}$ & $i \in\{\operatorname{car} 1, \operatorname{car} 2\}$ \\
\hline const-step & $\begin{array}{l}\mathscr{G}_{i}=C_{i}+\alpha E T_{i}+\beta E S D E_{i}\left(t_{d, i}\right)+\gamma E S D L_{i}\left(t_{d, i}\right), \\
\mathscr{G}_{P T}=\lambda T_{P T} 1_{\mathrm{PAY}}+K+(\alpha+\pi) T_{P T} \\
t_{0}=t_{d, R P}+\bar{T}_{R P} \\
E S D E_{i}\left(t_{d, i}\right)=\left(t_{0}-t_{d, i}-E T_{i}\right) F_{X_{i}}\left(t_{0}-t_{d, i}-E T_{i}\right)-\int_{-\infty}^{t_{0}-t_{d, i}-E T_{i}} x f_{X_{i}}(x) d x \\
E S D L_{i}\left(t_{d, i}\right)=\left(t_{d, i}+E T_{i}-t_{0}\right)\left(1-F_{X_{i}}\left(t_{0}-t_{d, i}-E T_{i}\right)\right)-\int_{t_{0}-t_{d, i}-E T_{i}} x f_{X_{i}}(x) d x\end{array}$ & $i \in\{\operatorname{car} 1, \operatorname{car} 2\}$ \\
\hline
\end{tabular}

$\dagger 1_{\mathrm{PAY}}$ : dummy variable, 0 if traveler has a travel card for public transport, 1 otherwise.

$F_{X_{i}}, f_{X_{i}}:$ cumulative distribution function/density function for $X_{i}=T_{i}-E T_{i}$.

$t_{d, R P}:$ RP departure time. $\bar{T}_{R P}$ : midpoint of usual travel time interval. 
specific $t_{0}$. To do this we make the assumption that $\bar{T}_{R P}$ is the travel time expected by the traveler and that $t_{d, R P}$ is the optimal departure time given a fixed travel time of $\bar{T}_{R P}$, i.e. $\frac{\partial G_{\mathrm{car}}}{\partial t_{d}}\left(t_{d, R P}, \bar{T}_{R P}, C\right)=0$. If $\bar{T}_{R P}$ is missing (because the respondent did not state such an interval), we use the respondent's actual arrival time instead. The resulting expressions for $t_{0}$ are also shown in Table 2, and the derivation of these expressions can be found in Appendix A.

To estimate the model parameters, we need to compute the expectations $E T, E e^{\eta T}$, $E T^{2}, E S D E$, and $E S D L$ for the car alternatives. However, since the travel time uncertainty in the SP exercises was presented to the respondents in terms of an interval, the travel time distribution is not uniquely defined. For this reason we have to make some assumption on the travel time distribution. For simplicity, we apply a Uniform travel time distribution, which allows us to compute the term $E e^{\eta T}$ analytically (as is required if using standard software to estimate the parameters). $7^{7}$

Recall that the travel time interval in the SP exercises was described as the interval of usual travel times, but that travel time approximately once a month would fall outside this interval. We model this by assuming a fixed probability $a$ with which travel time falls outside. We assume that the travel time distribution is symmetric around the interval midpoint, with probability mass $a / 2$ to the left of the interval and $a / 2$ to the right of the interval. Table 3 gives the resulting expressions for $E T, E e^{\eta T}, E T^{2}, E S D E$, and ESDL. The derivation can be found in Appendix $B$. When estimating the model parameters, we assume that $a=0.05$, which approximately corresponds to travel time falling outside the interval once a month if the trip is made once every weekday.

\footnotetext{
${ }^{7}$ As a sensitivity analysis, we also applied the Normal distribution, which gave roughly the same result. Another type of distribution yielding closed-form expressions for the generalized costs is a (shifted) Exponential distribution. However, the exponential distribution is not suitable because it assigns extremely large probabilities to travel times close to the minimum.
} 
Since the marginal effect of monetary cost $C$ on $\mathscr{G}_{i}$ is normalized to one, the estimated parameters are easy to interpret as $W_{0}, m, n, \zeta, \alpha, \beta, \gamma$, and $\pi$ have the unit SEK/minute, $\omega$ has the unit SEK/minute ${ }^{2}$, and $K$ has the unit SEK. The unit of the parameter $\eta$ is minute ${ }^{-1}$.

\subsection{Specification test}

Because of the large range in which the term $E e^{\eta T}$ may take values (from zero to very high values for some travel time distributions) we tested the model for misspecifications by checking the model's prediction power over the entire range of the RP travel time $T_{R P}$ stated by the respondents (which is correlated with $E e^{\eta T}$ ). We compared the predicted choice probabilities with the observed probabilities to calculate the prediction error, i.e. the absolute difference between the predicted and observed choice probabilities plotted against $T_{R P}$ (not shown here). We found no systematic relation between prediction error and travel time, indicating no systematic misspecification in this direction. Following Ben-Akiva and Lerman (1985), we also compared predicted and observed 'market shares' (i.e. sums of choice probabilities over individuals) separately for the three groups defined by $T_{R P}$ being less than 30 minutes, between 30 and 60 minutes, and above 60 minutes. Again we found no signs of systematic misspecification. 
Table 3: Assumed travel time distribution and the expectations $E e^{\eta T}, E T^{2}, E S D E$ and ESDL

Assumptions of travel time distribution

$T$ uniform

$E T=\bar{T}$

$\operatorname{Prob}\left(T<T^{\min }\right)=\frac{a}{2}$

$\operatorname{Prob}\left(T>T^{\max }\right)=\frac{a}{2}$

Implied expectations $E e^{\eta T}, E T^{2}, E S D E, E S D L$

$$
\begin{aligned}
& E e^{\eta T}=\frac{1}{\eta} \frac{1-a}{2 s} e^{\eta \bar{T}}\left(e^{\eta \frac{s}{1-a}}-e^{-\eta \frac{s}{1-a}}\right) \\
& E T^{2}=\bar{T}^{2}+\frac{s^{2}}{3(1-a)^{2}} \\
& E S D E= \begin{cases}0 & \text { if }\left(t_{0}-t_{d}-\bar{T}\right)<-\frac{s}{1-a}, \\
\frac{1-a}{4 s}\left(t_{0}-t_{d}-\bar{T}+\frac{s}{1-a}\right)^{2} & \text { if }-\frac{s}{1-a}<\left(t_{0}-t_{d}-\bar{T}\right)<\frac{s}{1-a}, \\
t_{0}-t_{d}-\bar{T} & \text { if }\left(t_{0}-t_{d}-\bar{T}\right)>\frac{s}{1-a} .\end{cases} \\
& E S D L= \begin{cases}t_{d}+\bar{T}-t_{0} & \text { if }\left(t_{0}-t_{d}-\bar{T}\right)<-\frac{s}{1-a}, \\
\frac{1-a}{4 s}\left(t_{0}-t_{d}-\bar{T}-\frac{s}{1-a}\right)^{2} & \text { if }-\frac{s}{1-a}<\left(t_{0}-t_{d}-\bar{T}\right)<\frac{s}{1-a}, \\
0 & \text { if }\left(t_{0}-t_{d}-\bar{T}\right)>\frac{s}{1-a} .\end{cases}
\end{aligned}
$$

Note: $T^{\min }, T^{\max }$ are the lower and upper endpoints of the travel time interval in the SP choice.

$\bar{T}$ is the midpoint and $s$ is half the interval width. 


\section{Results}

Table 4 presents log-likelihood values at convergence and goodness-of-fit statistics for all models (i.e. all combinations of preference specification and choice model specification). We estimated separate models for the full sample and for the subsamples of individuals having/not having a fixed work time. Estimations were carried out using Python Biogeme (see biogeme.epfl.ch and Bierlaire, 2003). The parameter estimates for the models with multiplicative errors are shown in Table 5, while Figures 36 display the resulting marginal utility functions $H$ and $W$, computed at the point estimates of the parameters and evaluated at $t_{0}=0$. Note that the Figures do not include confidence bands: They are used to illustrate the functional forms of $H$ and $W$ and illustrate differences between subsamples without taking into account whether these differences are significant. To be able to compare across subsamples and preference specifications, we present parameter estimates and the resulting $H$ and $W$ functions for models with multiplicative errors only. 8

\subsection{Can we estimate exponential preferences?}

A general finding for the models with exponential preference specifications (exp-exp and const-exp) is that the empirical parameter identification is very weak. This problem is worse for the models with additive errors than for models with multiplicative errors, as some models with additive errors have problems converging to a solution. A likely cause is that the term $E e^{\eta T}$ in the generalized costs $\mathscr{G}_{i}$ can become very large. This problem is smaller in the models with multiplicative errors, where the choice probabilities are functions of $\ln \left(\mathscr{G}_{i}\right)$.

However, even with multiplicative errors, parameter identification is weak as the

\footnotetext{
${ }^{8}$ Results for the models with additive errors are available on request.
} 
estimates of the parameters of $H$ and $W$ have very high standard errors and are highly correlated. In particular, we do not obtain meaningful results for the exp-exp specification with the sample with flexible work hours, where standard errors are unreasonably large. We report the results anyway, for completeness, but will not use them in the following. We conclude that, with our data, models with exponential preferences are hard to estimate.

For the const-affine and const-step preference specifications, we do not have similar problems with parameter identification. Here, the models with multiplicative errors tend to outperform models with additive errors (in terms of log-likelihood values).

\subsection{Performance of preference specifications}

We use the Akaike Information Criterion (AIC) and the Bayesian Information Criterion (BIC) to compare the preference specifications.$^{9}$ Both the AIC and the BIC are defined such that lower values indicate better fit (see e.g. Burnham and Anderson, 2004, for an interpretation).

The key issue is how the specifications with the additivity property (const-exp and const-affine) perform compared to the the const-step specification and to the more general exp-exp specification. Table 4 presents the following pattern:

1. When we model the behavior of non-flexible respondents (fixed work times), the const-step model gives a better fit than the exp-exp, const-exp and const-affine models. When we consider respondents with a more flexible schedule (flexible work times), the const-step model gives a worse fit than the other models. This is

\footnotetext{
${ }^{9}$ Letting $L L$ denote the optimal value of the log-likelihood function of a specific model, $\kappa$ the number of parameters, and $N$ the number of observations, the AIC and BIC are defined as AIC $=-2 L L+2 \kappa$ and $B I C=-2 L L+\kappa \log N$.
} 
consistent with theory, as the const-step model assumes a larger scheduling cost of arriving after the fixed preferred arrival time compared to arriving on time or shortly before. For the full sample, it is ambiguous which specification is better (the AIC and BIC disagree), but the differences between the specifications are small.

2. According to the AIC ranking, there is no clear pattern regarding the performance of models with the additivity property compared to the two benchmark models (the exp-exp and the const-step). According to the BIC, the const-affine model performs rather well: It is either the best specification, or very close to the best specification (in terms of fit).

\subsection{Estimates}

As stated, the empirical identification of the complex exponential preference specifications (exp-exp and const-exp) is weak: The estimates of the parameters of interest, $n, m$, $\eta$ and $W_{0}$, have very large standard errors (Table 5), and are highly correlated (the offdiagonal entries in the covariance matrix are numerically large). Nonetheless, the signs seem consistent across the different estimation samples: $W_{0}$ is negative, while $m$ and $\eta$ are positive. This pattern corresponds to the function $W$ being increasing and convex as shown in Figure 3. In the exp-exp specification, $n$ is always negative, corresponding to the function $H$ being decreasing and concave. Hence, $H$ and $W$ evaluated at the point estimates behave according to theory. This is also the case if we compute $W^{\prime}\left(t_{0}\right)$, the slope of $W$ at its intersection with $H$, at the point estimates (cf. Table 7): It is positive in accordance with theory. However, if we use the asymptotic distribution of the parameter estimates to simulate $W^{\prime}\left(t_{0}\right)$, we get the opposite result: $W^{\prime}\left(t_{0}\right)$ is negative, corresponding to $W$ being downwards sloping. The negative sign is not significant, however, as 
Table 4: Estimation Summary

\begin{tabular}{|c|c|c|c|c|c|c|c|c|c|}
\hline \multirow[b]{2}{*}{ Sample } & \multirow[b]{2}{*}{ Specification } & \multirow[b]{2}{*}{$\mathrm{N}$ par } & \multirow[b]{2}{*}{$\mathrm{N}$ obs } & \multicolumn{3}{|c|}{ Additive errors } & \multicolumn{3}{|c|}{ Multiplicative errors } \\
\hline & & & & LL & AIC & $\mathrm{BIC}$ & LL & AIC & $\mathrm{BIC}$ \\
\hline All & exp-exp & 8 & 1547 & no conv & - & - & -1283.54 & 2583.08 & 2625.83 \\
\hline All & const-exp & 7 & 1547 & no conv & - & - & -1286.91 & 2587.83 & 2625.24 \\
\hline All & const-affine & 6 & 1547 & -1293.28 & 2598.57 & 2630.63 & -1287.28 & 2586.57 & 2618.63 \\
\hline All & const-step & 7 & 1547 & -1288.13 & 2590.26 & 2627.67 & -1284.07 & 2582.14 & 2619.55 \\
\hline Fixed work time & exp-exp & 8 & 528 & no conv & - & - & -425.00 & 866.00 & 900.15 \\
\hline Fixed work time & const-exp & 7 & 528 & no conv & - & - & -426.63 & 867.25 & 897.14 \\
\hline Fixed work time & const-affine & 6 & 528 & -440.22 & 892.44 & 918.06 & -426.74 & 865.48 & 891.10 \\
\hline Fixed work time & const-step & 7 & 528 & -430.47 & 874.93 & 904.82 & -422.69 & 859.37 & 889.25 \\
\hline Flex work time & exp-exp & 8 & 1019 & -827.86 & 1671.72 & 1711.13 & -825.42 & 1666.84 & 1706.26 \\
\hline Flex work time & const-exp & 7 & 1019 & -828.59 & 1671.18 & 1705.67 & -828.14 & 1670.27 & 1704.76 \\
\hline Flex work time & const-affine & 6 & 1019 & -828.71 & 1669.43 & 1698.99 & -828.37 & 1668.75 & 1698.31 \\
\hline Flex work time & const-step & 7 & 1019 & -830.10 & 1674.19 & 1708.68 & -830.27 & 1674.54 & 1709.03 \\
\hline
\end{tabular}

LL: Log likelihood value at convergence

AIC: Akaike information criterion

BIC: Bayesian information criterion 
Table 5: Parameter estimates with robust standard errors (models with multiplicative error terms).

\begin{tabular}{|c|c|c|c|c|c|c|c|c|c|}
\hline \multirow{4}{*}{$\begin{array}{l}\text { Specification } \\
\text { Sample } \\
\begin{array}{l}\text { Parameter } \\
\mu \text { (error scale) }\end{array}\end{array}$} & \multirow{2}{*}{\multicolumn{3}{|c|}{$\begin{array}{c}\text { Exp-Exp } \\
\text { All }\end{array}$}} & \multicolumn{3}{|c|}{ Exp-Exp } & \multicolumn{3}{|c|}{ Exp-Exp } \\
\hline & & & & \multirow{3}{*}{$\begin{array}{c}\text { Estimate } \\
1.41\end{array}$} & work time & & Fle & $\mathrm{x}$ work time & \\
\hline & \multirow{2}{*}{$\begin{array}{c}\text { Estimate } \\
2.64\end{array}$} & \multicolumn{2}{|l|}{ Std Err } & & \multicolumn{2}{|l|}{ Std Err } & \multirow{2}{*}{$\begin{array}{c}\text { Estimate } \\
3.48\end{array}$} & \multicolumn{2}{|l|}{ Std Err } \\
\hline & & 0.294 & $* * *$ & & 0.554 & ** & & 0.513 & *** \\
\hline$n$ & -3.40 & 5.35 & & -2.19 & 1.71 & & -7.19 & 277 & \\
\hline$m$ & 6.28 & 9.99 & & 5.69 & 7.19 & & 7.03 & 277 & \\
\hline$\eta \cdot 1000$ & 2.87 & 4.52 & & 10.0 & 13.1 & & 1.28 & 49.6 & \\
\hline$W_{0}$ & -5.30 & 9.98 & & -5.78 & 6.80 & & -5.78 & 277 & \\
\hline$K$ & 36.9 & 11.7 & *** & 14.4 & 15.3 & & 47.3 & 12.9 & *** \\
\hline$\lambda$ & 1.56 & 0.301 & *** & 2.41 & 1.45 & $*$ & 1.29 & 0.297 & *** \\
\hline$\pi$ & -0.510 & 0.263 & * & 0.227 & 0.441 & & -0.712 & 0.288 & $* *$ \\
\hline Specification & \multicolumn{3}{|c|}{ Const-Exp } & \multicolumn{3}{|c|}{ Const-Exp } & \multicolumn{3}{|c|}{ Const-Exp } \\
\hline Sample & \multicolumn{3}{|c|}{ All } & \multicolumn{3}{|c|}{ Fixed work time } & \multicolumn{3}{|c|}{ Flex work time } \\
\hline Parameter & Estimate & Std Err & & Estimate & Std Err & & Estimate & Std Err & \\
\hline$\mu$ (error scale) & 2.59 & 0.289 & *** & 1.56 & 0.465 & $* * *$ & 3.38 & 0.353 & $* * *$ \\
\hline$m$ & 5.58 & 5.47 & & 16.1 & 32.2 & & 3.12 & 4.14 & \\
\hline$\eta \cdot 1000$ & 4.55 & 4.48 & & 4.37 & 9.38 & & 5.03 & 6.53 & \\
\hline$W_{0}$ & -4.45 & 5.45 & & -15.8 & 32.1 & & -1.77 & 4.13 & \\
\hline K & 32.8 & 11.4 & *** & 6.75 & 13.6 & & 43.4 & 12.0 & $* * *$ \\
\hline$\lambda$ & 1.61 & 0.318 & *** & 2.27 & 1.11 & $* *$ & 1.32 & 0.284 & $* * *$ \\
\hline$\pi$ & -0.372 & 0.245 & & 0.442 & 0.407 & & -0.588 & 0.252 & $* *$ \\
\hline Specification & \multicolumn{3}{|c|}{ Const-Affine } & \multicolumn{3}{|c|}{ Const-Affine } & \multicolumn{3}{|c|}{ Const-Affine } \\
\hline Sample & \multicolumn{3}{|c|}{ All } & \multicolumn{3}{|c|}{ Fixed work time } & \multicolumn{3}{|c|}{ Flex work time } \\
\hline Parameter & Estimate & Std Err & & Estimate & Std Err & & Estimate & Std Err & \\
\hline$\mu$ (error scale) & 2.64 & 0.279 & *** & 1.63 & 0.402 & $* * *$ & 3.42 & 0.346 & $* * *$ \\
\hline$\omega$ & 0.0252 & 0.00351 & $* * *$ & 0.0688 & 0.0197 & $* * *$ & 0.0157 & 0.00237 & $* * *$ \\
\hline$\zeta$ & 1.16 & 0.112 & *** & 0.423 & 0.236 & $*$ & 1.37 & 0.121 & $* * *$ \\
\hline K & 33.1 & 11.35 & *** & 7.24 & 13.8 & & 43.5 & 12.0 & *** \\
\hline$\lambda$ & 1.59 & 0.305 & *** & 2.17 & 0.914 & $* *$ & 1.31 & 0.278 & *** \\
\hline$\pi$ & -0.384 & 0.243 & & 0.410 & 0.398 & & -0.596 & 0.251 & $* *$ \\
\hline Specification & \multicolumn{3}{|c|}{ Const-Step } & \multicolumn{3}{|c|}{ Const-Step } & & onst-Step & \\
\hline Sample & & All & & Fixed & work time & & & $\mathrm{x}$ work time & \\
\hline Parameter & Estimate & Std Err & & Estimate & Std Err & & Estimate & Std Err & \\
\hline$\mu$ (error scale) & 2.96 & 0.303 & *** & $2.18 \quad 27$ & 0.434 & *** & 3.64 & 0.367 & *** \\
\hline$\alpha$ & 1.01 & 0.1070 & *** & 0.362 & 0.212 & $*$ & 1.26 & 0.1125 & $* * *$ \\
\hline$\beta$ & 0.555 & 0.0805 & $* * *$ & 1.15 & 0.241 & $* * *$ & 0.365 & 0.0696 & $* * *$ \\
\hline$\gamma$ & 0.853 & 0.123 & ${ }^{* * *}$ & 1.75 & 0.409 & $* * *$ & 0.540 & 0.0936 & $* * *$ \\
\hline$K$ & 39.4 & 10.77 & *** & 19.2 & 16.8 & & 46.8 & 11.35 & *** \\
\hline$\lambda$ & 1.40 & 0.253 & *** & 1.63 & 0.539 & $* * *$ & 1.22 & 0.250 & *** \\
\hline$\pi$ & -0.330 & 0.226 & & 0.407 & 0.400 & & -0.545 & 0.235 & $* *$ \\
\hline
\end{tabular}

*** denotes significance at the $1 \%$ level, $* *$ at the $5 \%$ level and * at the $10 \%$ level. 
Table 6: Value of travel time in SEK/hour, simulated mean with $95 \%$ confidence bands (models with multiplicative error terms)

\begin{tabular}{llccc}
\hline Specification & Mode & All & Fixed work time & Flex work time \\
\hline const-exp & car & $68[55 ; 81]$ & $22[-10 ; 56]$ & $81[67 ; 95]$ \\
const-exp & PT & $46[17 ; 74]$ & $49[0 ; 98]$ & $46[17 ; 75]$ \\
const-affine & car & $69[56 ; 83]$ & $25[-3 ; 53]$ & $82[68 ; 96]$ \\
const-affine & PT & $46[18 ; 75]$ & $50[1 ; 98]$ & $47[18 ; 75]$ \\
const-step & car & $61[48 ; 73]$ & $22[-3 ; 47]$ & $76[62 ; 89]$ \\
const-step & PT & $41[15 ; 67]$ & $46[2 ; 90]$ & $43[16 ; 70]$ \\
\hline
\end{tabular}

Table 7: $W^{\prime}\left(t_{0}\right)$ at point estimates and simulated mean $W^{\prime}\left(t_{0}\right)$ with $95 \%$ confidence bands (models with multiplicative error terms)

\begin{tabular}{|c|c|c|c|c|c|c|}
\hline \multirow[b]{2}{*}{ Specification } & \multicolumn{2}{|r|}{ All } & \multicolumn{2}{|c|}{ Fixed work time } & \multicolumn{2}{|c|}{ Flex work time } \\
\hline & $\begin{array}{l}\text { At point } \\
\text { estimates }\end{array}$ & $\begin{array}{c}\text { Simulated } \\
\text { (with conf lim) }\end{array}$ & $\begin{array}{l}\text { At point } \\
\text { estimates }\end{array}$ & $\begin{array}{c}\text { Simulated } \\
\text { (with conf lim) }\end{array}$ & $\begin{array}{l}\text { At point } \\
\text { estimates }\end{array}$ & $\begin{array}{c}\text { Simulated } \\
\text { (with conf lim) }\end{array}$ \\
\hline exp-exp & 0.018 & $-0.026[-0.21 ; 0.022]$ & 0.057 & $-0.035[-0.42 ; 0.079]$ & 0.0090 & $-13[-68 ;-0.0041]$ \\
\hline const-exp & 0.025 & $0.0013[-0.10 ; 0.030]$ & 0.070 & $-0.23[-1.4 ; 0.090]$ & 0.016 & $-0.011[-0.12 ; 0.018]$ \\
\hline const-affine & 0.025 & $0.025[0.018 ; 0.032]$ & 0.069 & $0.069[0.030 ; 0.11]$ & 0.016 & $0.016[0.011 ; 0.020]$ \\
\hline
\end{tabular}


shown in Table $7^{10} 11$

As indicated by Figures 3 ,4, there may be considerable differences between the samples. Judging from the Figures, the subsample with fixed work times has a steeper $W$ function than the other samples, which is expected since they face larger scheduling costs of late arrival. This is confirmed by the measure of $W^{\prime}\left(t_{0}\right)$ evaluated at the point estimates (cf. Table 7), but the effect is not significant.

Turning to the const-affine and const-step preference specifications, the parameters of interest $\omega, \zeta, \alpha, \beta$ and $\gamma$ all have the expected sign (positive) and are significant at the $10 \%$ level, most also at the $1 \%$ level. In the const-affine case, this corresponds to the affine $W$ functions having positive slopes and positive intercepts, in accordance with theory. Again the subsample with fixed work times has a steeper $W$ than the other samples (cf. Figure 5). The difference between the fixed and flexible work times subsamples is significant at the $5 \%$ level, while the difference between the fixed work times subsample and the full sample is marginally significant (Table 7). We find the same pattern in the const-step case, where the subsample with fixed work times has a much larger jump in $W$ than the other samples, and a higher value of $W$ after $t_{0}$ (cf. Figure 6).

\footnotetext{
${ }^{10}$ This does not hold for the exp-exp specification with the sample with flexible work hours, but as already mentioned, we did not obtain reliable results for this model.

${ }^{11}$ We also simulated the distributions for $H$ and $W$ (point-wise) using draws from the asymptotic distribution of the parameters (results not shown here): In the exp-exp and const-exp specifications, the simulated mean of $W$ is decreasing in $t$, and in the exp-exp specification, the simulated mean of $H$ is either increasing in $t$ or non-monotone. This is most likely a consequence of the large standard errors, the high correlation between parameter estimates, and the exponential formulation, which is very sensitive to the large parameter values that may arise when drawing from normal distributions. Note that both the point estimates of $H$ and $W$ and the simulated means lie within the simulated 95\% confidence bands, i.e. the standard errors are too large to say with certainty whether the functions are increasing or decreasing. Again, this does not hold for the exp-exp specification with the sample with flexible work hours, which we omitted from the analysis.
} 
Interpretation of the PT-specific parameters $K$ and $\pi$ should be done with some caution, as there are potential sources of bias. First, the parameter $K$ picks up mode preferences and other such effects that are not explicitly modeled, as the average additional cost stemming from time spent walking to/from the station/stop or waiting at the station/stop (we consider only the total public transport travel time and do not distinguish between different components of travel time). Second, if $\lambda T$ is a poor approximation of the monetary cost of the PT alternative, such that we systematically under-/overestimate the monetary cost, $K$ and $\pi$ may be biased.

Table 6 shows the values of travel time for car and public transport for all samples and preference specifications. The values of time are derived in Appendix C. In the exp-exp specification the value of time is a function of the given travel time distribution and therefore varies over choices. Its distribution in the data reflects not just preferences but also the experimental design, so we do not show it here. The values of time are similar across preference specifications which is reassuring. Note that the values of time for PT depend on $\pi$ and so should be interpreted with some caution (cf. above). For travelers with flexible working time the value of time is reasonable for commuters in Stockholm, maybe at the lower end. For travelers with fixed working time the value of time is even lower. One reason could be that the latter commuters are more sensitive to monetary costs, since they have lower average income than commuters with flexible working time. Moreover, the low values of time found in this study may also be an effect of a policy bias against congestion pricing; the data was collected just before the trial with congestion charges was introduced in Stockholm during an intense debate in the media.

The PT-specific constant $K$ is large and positive indicating that using public transport carries an additional fixed cost compared to using car for this sample of drivers. This 


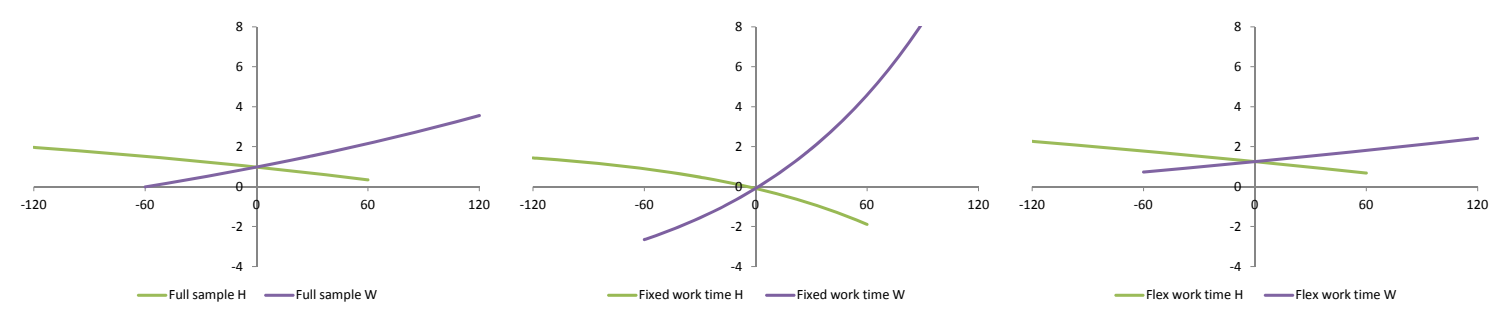

Figure 3: Estimated functions $H$ and $W$, evaluated at $t_{0}=0$, exp-exp model with multiplicative error terms

constant is large in particular for commuters with flexible work time, 40-50 SEK. As mentioned above, the parameter $K$ should be interpreted with some caution, but it seems plausible that car commuters in the congested morning peak have reasons for preferring car to public transport and therefore have a tendency to stick to car.

The positive signs of $\lambda$ are in accordance with our expectation, indicating that the monetary cost of the public transport alternative is positively related to the travel time. The parameter $\pi$ (the difference between the value of travel time in PT and car) is not significantly positive in any of the models. For the subsample with fixed work times, it is positive but insignificant, while it is significantly negative (5\% level) for the subsample with flexible work times. For the full sample, $\pi$ is negative but rarely significantly. The negative sign indicates that the marginal utility of travel time in public transport exceeds the marginal utility of car travel time. This could be the case if travel time spent in the public transport (primarily the Metro) is more comfortable (it is possible to read or sleep) or productive than time spent driving in the morning peak. However, as mentioned above, the parameter $\pi$ should be interpreted with caution. 

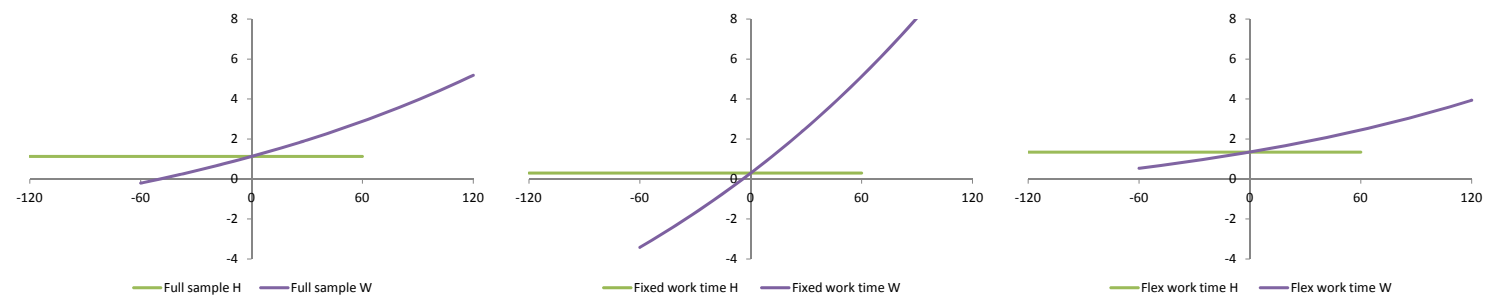

Figure 4: Estimated functions $H$ and $W$, evaluated at $t_{0}=0$, const-exp model with multiplicative error terms
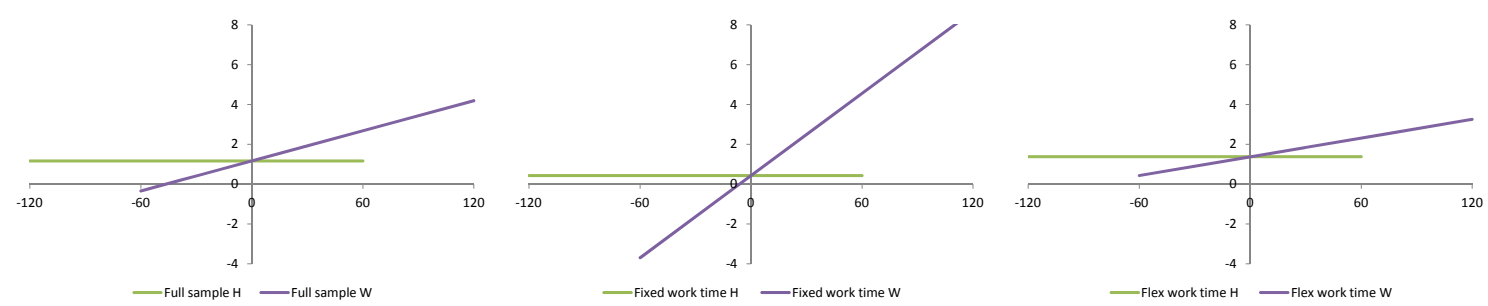

Figure 5: Estimated functions $H$ and $W$, evaluated at $t_{0}=0$, const-affine model with multiplicative error terms
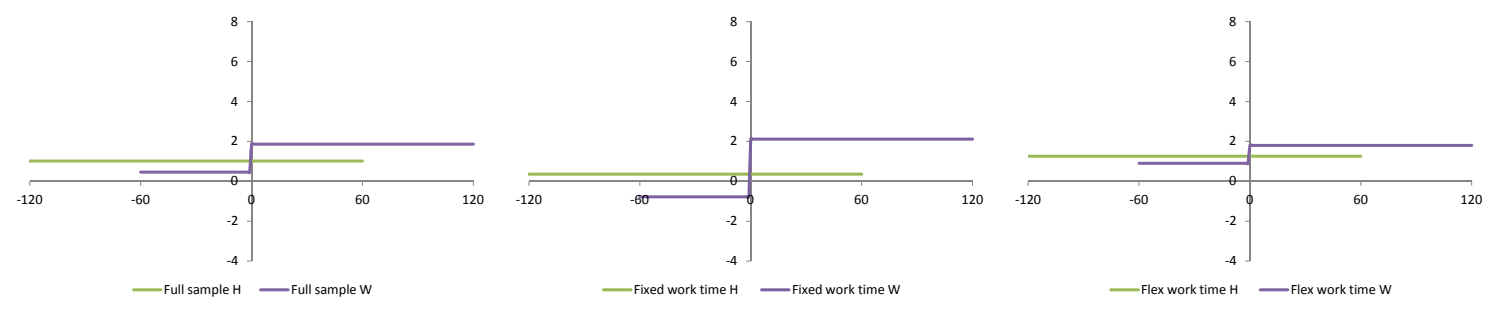

Figure 6: Estimated functions $H$ and $W$, evaluated at $t_{0}=0$, const-step model with multiplicative error terms 


\subsection{Robustness check}

A relevant concern in our analysis is that the PT alternative differs from the car alternatives in several ways: First, PT travel time and departure time are stated by the respondent and not given in the questionnaire like the car attributes are. Moreover, the actual values are not displayed in the SP choice (cf. Figure 2). And finally, the monetary cost is unknown and has to be approximated. All this may cause some sort of 'measurement error' when comparing alternatives, resulting in a different error scale for the PT alternative. Since we assume a common error scale for all alternatives, this would bias our estimated parameters. Another issue is whether excluding the 'Bike/Walk' and 'Cancel the trip' alternatives and keeping the PT alternative leads to biased results. 12

As a robustness check, we re-estimated the model using a nested logit structure, with the two car alternatives in one nest and the PT alternative in another. This nested logit model allows us to capture some un-modeled similarities of the two car alternatives in a common error scale that differs from that of the PT alternative. The nested logit specification yields the same ranking in terms of AIC and BIC as the model above, which we believe adds credibility to our results. The gain in log-likelihood from using nested logit instead of logit is very modest however, always less than 0.75 units.

As a further robustness check, we investigated the effect of our choice to omit the 'Bike/Walk' and 'Cancel the trip' alternatives and keep the PT alternative. We estimated the following two models:

A) A model which includes all five alternatives: The generalized cost of alternatives 'Bike/Walk' and 'Cancel the trip' is constant (modeled as alternative-specific constants).

\footnotetext{
${ }^{12} \mathrm{We}$ thank two anonymous referees for raising these issues and suggesting the reported robustness checks.
} 
B) A model which includes only the two car alternatives (omitting the PT alternative from the current model), estimated on the subset of choices where one of the car alternatives is chosen.

Model A) yields the same ranking as the model in section 4. It yields slightly different estimates of the parameters, but these differences are not significant (judging from the $95 \%$ confidence intervals).

Model B) yields small differences in model ranking; the most important being that the ranking changes for the full sample such that the const-affine specification is now best and const-step worst. This does not affect our conclusions, as the pattern for the full sample was ambiguous to begin with. Our conclusions remain the same: For the subsample with fixed work hours, the const-step specification performs better than the other specifications, or at least close to equally well as the best of the other specifications. For the subsample with flexible work hours, the const-step specification performs worse than the other specifications, and the const-affine specification is best. Overall, in terms of the BIC, the const-affine specification performs better than or equally well as the other specifications.

Model B) also yields slightly different estimates of parameters in $\mathrm{H}$ and $\mathrm{W}$, but again the estimates are not significantly different from those in Table 5 (judged by comparing 95\% confidence intervals).

The results of the nested logit models and models A and B can be seen in details in Appendix D. 


\section{Conclusion}

The only form of smooth scheduling preferences with a non-trivial value of travel time variability which provides travel costs that are additive over links in transport networks where link travel times are arbitrarily distributed independent random variables, is a constant marginal utility of time at origin $(H)$ and an exponential marginal utility at the destination $(W)$. This const-exp formulation includes the const-affine specification as a limiting case. The purpose of this paper has been to empirically estimate these scheduling functions, to reveal the methodological issues involved, and to compare them both to a more general form (exp-exp formulation) and to a conventional form (conststep) with regard to model fit. We used SP data collected from car commuters traveling in the morning peak into the city of Stockholm.

An important limitation of our analysis (and of any analysis we know of) is that we had to make some strong assumptions regarding $t_{0}$, the location in time where $H$ and $W$ intersect. The problem with obtaining a valid $t_{0}$ is that it is a somewhat artificial concept (the point in time where one would depart if travel time was zero with certainty), so asking respondents directly may not be meaningful. This implies that $t_{0}$ has to be assumed or somehow inferred from the background information about the respondents' journeys. In our case, we have inferred a value of $t_{0}$ from information about the respondents' usual travel time (expressed as an interval) and usual departure time - assuming that this departure time is optimally chosen given the scheduling model under consideration. Our assumption about $t_{0}$ may have biased the results, but we are not aware of other practically feasible formulations that yield better model fit (in terms of loglikelihood) 13 We believe more evidence is needed regarding how to approximate $t_{0}$ in a

\footnotetext{
${ }^{13}$ Our first attempt was to derive $t_{0}$ from information respondents stated about their travel time and preferred departure time under free flow conditions ('Had there been no queues on the road, what would
} 
choice model without strict behavioural assumptions, based on questions that are easily understood by respondents and hence trustworthy.

Our analysis shows that all the above mentioned scheduling specifications can be estimated, but that the parameters in the most complex models (exp-exp and const-exp) are hard to identify empirically. Firstly, we have difficulties with achieving convergence when the generalized cost function becomes complex and can take very large values, as is the case with the exponential specifications. Secondly, when we achieve convergence, the parameters tend to be very poorly identified, with large standard errors and large correlations of the parameter estimates. It may be possible to design an SP experiment that yields better identification of $H$ and $W$ in these complex scheduling models, but we are not aware of any SP experiments designed specifically for this; instead we applied a dataset with two features we guessed would help identification of our complex model specifications: Our data is based on an orthogonal design and has relatively large ranges of the departure time and travel time attributes 14 Yet we still experience empirical identification problems. We think it would be relevant to investigate these identification problems further, in particular examining which kind of SP design that would allow us to identify $H$ and $W$ more precisely, e.g. using synthetic data. We acknowledge the relevance of such an analysis, and consider it a potential issue for future research, but outside the scope of the current paper.

your travel time be?' and 'In case there were never any queues at any time of day, would you depart at a different time than what you actually did? If yes, when?'). That yields somewhat different parameter levels though the overall pattern is the same. We chose not to use this formulation because our current formulation has a much better fit (12-20 loglikelihood units better).

${ }^{14}$ As pointed out by Tseng and Verhoef (2008), the estimation of $H$ and $W$ demand very large ranges of departure times and travel times in the SP design. This is not so common: It is our impression that many studies tend to avoid very large ranges for fear that respondents would otherwise consider the SP tasks unrealistic. 
We used the Akaike Information Criterion (AIC) and the Bayesian Information Criterion (BIC) to compare all four specifications. According to the AIC ranking, there is no clear pattern regarding the performance of models with the additivity property compared to the two benchmark models (the exp-exp and the const-step). However, according to the BIC, the const-affine model performs rather well: It is either the best specification, or has similar fit as the best specification. The reason that the AIC and the BIC disagree is that the BIC penalizes the number of parameters harder than the AIC, particularly for larger sample sizes. Compared to the complex specifications with exponential marginal utilities that suffer from empirical identification problems, the constaffine specification has the benefit of being more parsimonious.

Not surprisingly, the difference in performance of the different scheduling models is related to the characteristics of the travelers in the analysis: For travelers with fixed working time the const-step specification has better fit than the other models, while the opposite is the case for the group of travelers with flexible working time. This is consistent with expectation, since the const-step model assumes a jump in scheduling costs when arriving even shortly after $t_{0}$ compared to arriving shortly before - which is what we would expect when people have fixed work start times.

In conclusion, our results for the exponential specifications are not decisive and so we are unable to judge whether the complex exponential specifications (const-exp and the exp-exp) are better than the simple const-affine specification. However, we can compare the const-affine and the conventional const-step specifications which both yield plausible results. Our results based on the BIC lend some support to the constaffine specification, in which the value of travel time variability is proportional to the variance of travel time. This is a nice and convenient result, as it lends support to using the variance of the travel time distribution as a measure of TTV, providing additive travel 
costs in transport networks with independent link travel times.

\section{Acknowledgements}

Maria Börjesson and Leonid Engelson have received financial support from the Centre for Transport Studies, Royal Institute of Technology, Sweden. Katrine Hjorth and Mogens Fosgerau have received financial support from the Danish Strategic Research Council. 


\section{References}

Abkowitz, M. D. (1981). An analysis of the commuter departure time decision. Transportation 10(3), 283-297.

Bates, J., J. Polak, P. Jones, and A. Cook (2001). The valuation of reliability for personal travel. Transportation Research, Part E 37(2-3), 191-229.

Ben-Akiva, M. and S. Lerman (1985). Discrete Choice Analysis. MIT Press, Cambridge, MA.

Benezech, V. and N. Coulombel (2013). The value of service reliability. Transportation Research, Part B 58, 1-15.

Bierlaire, M. (2003). Biogeme: A free package for the estimation of discrete choice models. Proceedings of the 3rd Swiss Transportation Research Conference. www.strc.ch/Paper/bierlaire.pdf.

Börjesson, M. (2008). Joint RP-SP data in a mixed logit analysis of trip timing decisions. Transportation Research, Part E 44(6), 1025-1038.

Börjesson, M. (2009). Modelling the preference for scheduled and unexpected delays. Journal of Choice Modelling 2, 29-50.

Börjesson, M., J. Eliasson, and J. Franklin (2012). Valuations of travel time variability in scheduling versus mean-variance models. Transportation Research, Part B 46(7), 855-873.

Burnham, K. P. and D. R. Anderson (2004). Multimodel inference - understanding AIC and BIC in model selection. Sociological Methods and Research 33(2), 261-304. 
Carrion, C. and D. Levinson (2012). Value of travel time reliability: A review of current evidence. Transportation Research Part A 46(4), 720-741.

de Jong, G., A. Daly, M. Pieters, C. Vellay, M. Bradley, and F. Hofman (2003). A model for time of day and mode choice using error components logit. Transportation Research, Part E 39(3), 245-268.

Eliasson, J. (2007). The relationship between travel time variability and road congestion. 11th World Conference on Transport Research, trid.trb.org.

Engelson, L. (2011). Properties of expected travel cost function with uncertain travel time. Transportation Research Record: Journal of the Transportation Research Board 2254, 151-159.

Engelson, L. and M. Fosgerau (2011). Additive measures of travel time variability. Transportation Research, Part B 45(10), 1560-1571.

Ettema, D., O. Ashiru, and J. Polak (2004). Modeling timing and duration of activities and trips in response to road-pricing policies. Transportation Research Record: Journal of the Transportation Research Board 1894, 1-10.

Fosgerau, M. (2009). The marginal social cost of headway for a scheduled service. Transportation Research, Part B 43(8-9), 813-820.

Fosgerau, M. and M. Bierlaire (2009). Discrete choice models with multiplicative error terms. Transportation Research, Part B 43(5), 494-505.

Fosgerau, M. and L. Engelson (2011). The value of travel time variance. Transportation Research, Part B 45(1), 1-8. 
Fosgerau, M. and D. Fukuda (2012). Valuing travel time variability: Characteristics of the travel time distribution on an urban road. Transportation Research, Part C 24, 83-101.

Fosgerau, M. and A. Karlström (2010). The value of reliability. Transportation Research, Part B 44(1), 38 - 49.

Hendrickson, C. and E. Plank (1984). The flexibility of departure times for work trips. Transportation Research, Part A 18(1), 25-36.

Jenelius, E., L.-G. Mattsson, and D. Levinson (2011). Traveler delay costs and value of time with trip chains, flexible activity scheduling and information. Transportation Research, Part B 45(5), 789-807.

Lam, W., H. K. Lo, and S. Wong (2014). Advances in equilibrium models for analyzing transportation network reliability. Transportation Research, Part B 66, 1-3.

Noland, R. B. and K. Small (1995). Travel-time uncertainty, departure time choice, and the cost of morning commutes. Transportation Research Record 1493, 150-158.

Small, K. (1982). The scheduling of consumer activities: Work trips. The American Economic Review 72(3), 467-479.

Tseng, Y.-Y. and E. T. Verhoef (2008). Value of time by time of day: A stated-preference study. Transportation Research, Part B 42(7-8), 607-618.

Vickrey, W. S. (1969). Congestion theory and transport investment. The American Economic Review 59(2), 251-260.

Vickrey, W. S. (1973). Pricing, metering, and efficiently using urban transportation facilities. Highway Research Record 476, 36 - 48. 
Wooldridge, J. (2002). Econometric Analysis of Cross Section and Panel Data. MIT Press, Cambridge, MA. 


\section{A Derivation of generalized costs in Table 2}

\section{A.1 Car alternatives}

For the car alternatives, we assume that $\mathscr{G}_{i}$ is the expected generalized cost $E G_{\text {car }}\left(t_{d}, C\right)$ from the model in section 2 . The expectation is taken with respect to the travel time distribution. This is evaluated for the departure time and monetary cost given as alternative attributes in the questionnaire - hence departure time is not necessarily optimal.

For the exp-exp model, $\mathscr{G}_{i}$ is the expected value of (7). $\mathscr{G}_{i}$ for the const-exp model is the same, except that we set $n=0$. For the const-affine model and the const-step model, $\mathscr{G}_{i}$ is the expected value of (11) and (15), respectively.

To compute the expectations of SDE and SDL, we apply the decomposition $T=X+$ $E T$, where $X$ is a random variable with $E X=0$. Let $F_{X}$ and $f_{X}$ denote the cumulative distribution function and density function of $X$. Then ESDE and ESDL are given by:

$$
\begin{aligned}
\operatorname{EDSE}\left(t_{d}\right) & :=E\left(\max \left(t_{0}-t_{d}-E T-X, 0\right)\right) \\
& =\int_{-\infty}^{t_{0}-t_{d}-E T}\left(t_{0}-t_{d}-E T-x\right) f_{X}(x) d x \\
& =\left(t_{0}-t_{d}-E T\right) F_{X}\left(t_{0}-t_{d}-E T\right)-\int_{-\infty}^{t_{0}-t_{d}-E T} x f_{X}(x) d x \\
\operatorname{EDSL}\left(t_{d}\right) & :=E\left(\max \left(t_{d}+X+E T-t_{0}, 0\right)\right) \\
& =\int_{t_{0}-t_{d}-E T}^{\infty}\left(t_{d}+x+E T-t_{0}\right) f_{X}(x) d x \\
& =\left(t_{d}+E T-t_{0}\right)\left(1-F_{X}\left(t_{0}-t_{d}-E T\right)\right)+\int_{t_{0}-t_{d}-E T}^{\infty} x f_{X}(x) d x \\
& =\left(t_{d}+E T-t_{0}\right)\left(1-F_{X}\left(t_{0}-t_{d}-E T\right)\right)-\int_{-\infty}^{t_{0}-t_{d}-E T} x f_{X}(x) d x .
\end{aligned}
$$

With all four preference specifications, $\mathscr{G}_{i}$ depends on $t_{0}$, which is unobserved. We derive an approximation of the individual-specific $t_{0}$ by assuming that the respondent's $\mathrm{RP}$ departure time $\left(t_{d, R P}\right)$ is the optimal departure time for a trip with no travel time 
variability and travel time equal to $\left(\bar{T}_{R P}\right)$, the midpoint of the usual travel time interval stated by the respondent. If $\bar{T}_{R P}$ is missing (because the respondent did not state such an interval), we use the respondent's actual arrival time instead. The assumption that $t_{d, R P}$ is optimal given $\bar{T}_{R P}$ is equivalent to $\frac{\partial G_{\mathrm{car}}}{\partial t_{d}}\left(t_{d, R P}, \bar{T}_{R P}, C\right)=0$, which for the models with continuous $H$ and $W$ is equivalent to

$$
H\left(t_{d, R P}\right)=W\left(t_{d, R P}+\bar{T}_{R P}\right)
$$

i.e. that the MUT at origin at the chosen departure time equals the MUT at destination at the planned arrival time.

For the exp-exp model, we use (5) and (6) to see that (23) is equivalent to

$$
m-n+n e^{\eta\left(t_{d, R P}-t_{0}\right)}=m e^{\eta\left(t_{d, R P}+\bar{T}_{R P}-t_{0}\right)},
$$

which implies that

$$
t_{0}=t_{d, R P}+\frac{1}{\eta} \ln \left(\frac{m e^{\eta \bar{T}_{R P}}-n}{m-n}\right) .
$$

For the const-exp model (with $n=0$ ), this reduces to

$$
t_{0}=t_{d, R P}+\bar{T}_{R P}
$$

For the const-affine model, we use (9) and (10) to see that $(23)$ is equivalent to

$$
0=\omega\left(t_{d, R P}+\bar{T}_{R P}-t_{0}\right)
$$

which implies that

$$
t_{0}=t_{d, R P}+\bar{T}_{R P}
$$

In the const-step model, we use (13) and (14) to see that the assumption $\frac{\partial G_{\mathrm{car}}}{\partial t_{d}}\left(t_{d, R P}, \bar{T}_{R P}, C\right)=$ 0 is equivalent to

$$
t_{0}=t_{d, R P}+\bar{T}_{R P}
$$




\section{A.2 PT alternatives}

For the PT alternatives, we assume that $\mathscr{G}_{P T}$ is the generalized cost $G_{P T}\left(t_{d, P T}, C_{P T}\right)$ from the model in section 2, evaluated for a deterministic travel time of $T_{P T}$ and for the optimal departure time given $T_{P T}$. As discussed in section 4.2, the monetary cost is assumed equal to zero if the respondent holds a travel card and equal to $C_{P T}=\lambda T_{P T}$ otherwise ( $\lambda$ is a parameter to be estimated).

For the exp-exp model, optimizing (8) with respect to departure time yields

$$
t_{d, P T}=t_{0}-\frac{1}{\eta} \ln \left(\frac{m e^{\eta T_{P T}}-n}{m-n}\right),
$$

and inserting this back in (8) yields

$$
\mathscr{G}_{P T}=\lambda T_{P T} 1_{\mathrm{PAY}}+K+\left(W_{0}+\pi\right) T_{P T}+\frac{m-n}{\eta} \ln \left(\frac{m e^{\eta T_{P T}}-n}{m-n}\right),
$$

where $1_{\text {PAY }}$ is a dummy variable, which is 0 if the traveller has a travel card for public transport, and 1 otherwise.

We get $\mathscr{G}_{P T}$ for the const-exp model by setting $n=0$ in (24). For the const-affine model, optimizing (12) with respect to departure time yields

$$
t_{d, P T}=t_{0}-T_{P T}
$$

and inserting this back in (12) yields

$$
\mathscr{G}_{P T}=\lambda T_{P T} 1_{\mathrm{PAY}}+K+(\zeta+\pi) T_{P T}
$$

Similarly, for the const-step model, we optimize (16) with respect to departure time and get

$$
t_{d, P T}=t_{0}-T_{P T} .
$$

Inserting this back in (16) yields

$$
\mathscr{G}_{P T}=\lambda T_{P T} 1_{\mathrm{PAY}}+K+(\alpha+\pi) T_{P T} .
$$




\section{B Derivation of expectations in Table 3}

Let $T^{\min }$ and $T^{\max }$ denote the lower and upper endpoints of the travel time interval in the SP choice. $\bar{T}$ denotes the midpoint of the travel time interval, and $s$ half the interval width.

We assume that $T$ is uniform with $E T=\bar{T}$ and $\operatorname{Prob}\left(T<T^{\min }\right)=\operatorname{Prob}\left(T>T^{\max }\right)=$ $\frac{a}{2}$. This implies that the support of $T$ has width

$$
\frac{T^{\max }-T^{\min }}{1-a}=\frac{2 s}{1-a}
$$

and so the support is given by $\operatorname{supp}_{T}=\left[\bar{T}-\frac{s}{1-a} ; \bar{T}+\frac{s}{1-a}\right]$. Again, we apply the decomposition $T=X+E T$, where $X$ is a random variable with $E X=0$. When $T$ is uniformly distributed on $\left[\bar{T}-\frac{s}{1-a} ; \bar{T}+\frac{s}{1-a}\right], X$ is uniformly distributed on $\left[-\frac{s}{1-a} ; \frac{s}{1-a}\right]$ and has the density function

$$
f_{X}(x)= \begin{cases}\frac{1-a}{2 s} & \text { if }-\frac{s}{1-a}<x<\frac{s}{1-a} \\ 0 & \text { else }\end{cases}
$$

We use the density function to compute $E e^{\eta T}$ and $E T^{2}$ :

$$
\begin{aligned}
E e^{\eta T} & =e^{\eta \bar{T}} E e^{\eta X}=e^{\eta \bar{T}} \int_{-\infty}^{\infty} e^{\eta x} f_{X}(x) d x \\
& =\frac{1-a}{2 s} e^{\eta \bar{T}} \int_{-\frac{s}{1-a}}^{\frac{s}{1-a}} e^{\eta x} d x=\frac{1}{\eta} \frac{1-a}{2 s} e^{\eta \bar{T}}\left(e^{\eta \frac{s}{1-a}}-e^{-\eta \frac{s}{1-a}}\right) \\
E T^{2} & =\bar{T}^{2}+E X^{2}=\bar{T}^{2}+\int_{-\infty}^{\infty} x^{2} f_{X}(x) d x \\
& =\bar{T}^{2}+\frac{1-a}{2 s} \int_{-\frac{s}{1-a}}^{\frac{s}{1-a}} x^{2} d x=\bar{T}^{2}+\frac{s^{2}}{3(1-a)^{2}}
\end{aligned}
$$

To compute EDSE and ESDL, we use (21) and 22). Note that the cumulative dis- 
tribution function for $X$ is given by

$$
F_{X}(x)= \begin{cases}0 & \text { if } x<-\frac{s}{1-a}, \\ \frac{1-a}{2 s}\left(x+\frac{s}{1-a}\right) & \text { if }-\frac{s}{1-a}<x<\frac{s}{1-a} \\ 1 & \text { if } x>\frac{s}{1-a} .\end{cases}
$$

and that

$$
\int_{-\infty}^{z} x f_{X}(x) d x= \begin{cases}\frac{1-a}{4 s}\left(z^{2}-\left(\frac{s}{1-a}\right)^{2}\right) & \text { if }-\frac{s}{1-a}<x<\frac{s}{1-a} \\ 0 & \text { else. }\end{cases}
$$

Hence, it follows from (21) and 22) that ESDE and ESDL are

$$
\begin{gathered}
E S D E= \begin{cases}0 & \text { if }\left(t_{0}-t_{d}-\bar{T}\right)<-\frac{s}{1-a}, \\
\frac{1-a}{4 s}\left(t_{0}-t_{d}-\bar{T}+\frac{s}{1-a}\right)^{2} & \text { if }-\frac{s}{1-a}<\left(t_{0}-t_{d}-\bar{T}\right)<\frac{s}{1-a}, \\
t_{0}-t_{d}-\bar{T} & \text { if }\left(t_{0}-t_{d}-\bar{T}\right)>\frac{s}{1-a} .\end{cases} \\
E S D L= \begin{cases}t_{d}+\bar{T}-t_{0} & \text { if }\left(t_{0}-t_{d}-\bar{T}\right)<-\frac{s}{1-a}, \\
\frac{1-a}{4 s}\left(t_{0}-t_{d}-\bar{T}-\frac{s}{1-a}\right)^{2} & \text { if }-\frac{s}{1-a}<\left(t_{0}-t_{d}-\bar{T}\right)<\frac{s}{1-a}, \\
0 & \text { if }\left(t_{0}-t_{d}-\bar{T}\right)>\frac{s}{1-a} .\end{cases}
\end{gathered}
$$

\section{Derivation of values of travel time in Table 6}

To derive the values of travel time (VTT) for the models in section 2, we apply the decomposition $T=X+E T$, where $X$ is a random variable with $E X=0$. VTT is the derivative of the expected generalized cost for the optimal departure time with respect to $E T$.

For the exp-exp model, taking the expectation of (7) and (8) and setting the derivatives with respect to $t_{d}$ equal to zero leads to the following optimal departure time (for both car and PT):

$$
t_{d}^{*}=t_{0}-\frac{1}{\eta} \ln \left(\frac{m E e^{\eta T}-n}{m-n}\right)
$$


Inserting this into the expected generalized cost yields:

$$
\begin{aligned}
E G_{\mathrm{car}}^{*} & =C+W_{0} E T+\frac{m-n}{\eta} \ln \left(\frac{m E e^{\eta T}-n}{m-n}\right) \\
& =C+W_{0} E T+\frac{m-n}{\eta} \ln \left(\frac{m e^{\eta E T} E e^{\eta X}-n}{m-n}\right)
\end{aligned}
$$

and

$$
\begin{aligned}
E G_{P T}^{*} & =C_{P T}+K+\left(W_{0}+\pi\right) E T_{P T}+\frac{m-n}{\eta} \ln \left(\frac{m E e^{\eta T_{P T}}-n}{m-n}\right) \\
& =C_{P T}+K+\left(W_{0}+\pi\right) E T_{P T}+\frac{m-n}{\eta} \ln \left(\frac{m e^{\eta E T_{P T}} E e^{\eta X_{P T}}-n}{m-n}\right) .
\end{aligned}
$$

The value of travel time is

$$
\begin{aligned}
V T T_{\mathrm{car}} & =\frac{\partial E G_{\mathrm{car}}^{*}}{\partial E T}=W_{0}+(m-n) \frac{m e^{\eta E T} E e^{\eta X}}{m e^{\eta E T} E e^{\eta X}-n}, \\
V T T_{P T} & =\frac{\partial E G_{P T}^{*}}{\partial E T}=W_{0}+\pi+(m-n) \frac{m e^{\eta E T_{P T}} E e^{\eta X_{P T}}}{m e^{\eta E T_{P T} E} e^{\eta X_{P T}-n}} .
\end{aligned}
$$

So in the exp-exp model, the value of travel time varies over choices - it depends on both the mean and the shape of the travel time distribution.

For the const-exp model, the expressions for the optimal expected generalized cost reduce to

$$
\begin{aligned}
& E G_{\mathrm{car}}^{*}=C+\left(W_{0}+m\right) E T+\frac{m}{\eta} \ln \left(E e^{\eta X}\right), \\
& E G_{P T}^{*}=C_{P T}+K+\left(W_{0}+m+\pi\right) E T_{P T}+\frac{m}{\eta} \ln \left(E e^{\eta X_{P T}}\right),
\end{aligned}
$$

where the value of travel time is

$$
\begin{aligned}
& V T T_{\mathrm{car}}=W_{0}+m, \\
& V T T_{P T}=W_{0}+m+\pi .
\end{aligned}
$$

For the const-affine model, we take the expectation of (11) and (12). Setting the derivatives with respect to $t_{d}$ equal to zero leads to the following optimal departure time 
(for both car and PT):

$$
t_{d}^{*}=t_{0}-E T
$$

Inserting this into the expected generalized cost yields:

$$
E G_{\mathrm{car}}^{*}=C+\zeta E T+\frac{\omega}{2} \operatorname{var}(X)
$$

and

$$
E G_{P T}^{*}=C_{P T}+K+(\zeta+\pi) E T_{P T}+\frac{\omega}{2} \operatorname{var}\left(X_{P T}\right)
$$

So the value of travel time is

$$
\begin{aligned}
& V T T_{\text {car }}=\zeta, \\
& V T T_{P T}=\zeta+\pi .
\end{aligned}
$$

For the const-step model, we take the expectation of $(15)$ and $(16)$ and differentiate with respect to $t_{d}$. We use the expressions for ESDE and ESDL from (21) and (22). For both car and PT, the derivative is

$$
\frac{\partial E G}{\partial t_{d}}=-\beta F_{X}\left(t_{0}-t_{d}-E T\right)+\gamma\left(1-F_{X}\left(t_{0}-t_{d}-E T\right)\right),
$$

where $F_{X}$ is the cumulative distribution functions of $X$. Setting (25) equal to zero implies that the optimal departure time is

$$
t_{d}^{*}=t_{0}-E T-F_{X}^{-1}\left(\frac{\gamma}{\beta+\gamma}\right) .
$$

Inserting this into the expected generalized cost yields:

$$
E G_{\mathrm{car}}^{*}=C+\alpha E T+(\beta+\gamma) \int_{-\infty}^{F_{X}^{-1}\left(\frac{\gamma}{\beta+\gamma}\right)} x f_{X}(x) d x,
$$

and

$$
E G_{P T}^{*}=C_{P T}+K+(\alpha+\pi) E T_{P T}+(\beta+\gamma) \int_{-\infty}^{F_{X}^{-1}\left(\frac{\gamma}{\beta+\gamma}\right)} x f_{X_{P T}}(x) d x
$$


So the value of travel time is

$$
\begin{aligned}
& V T T_{\text {car }}=\alpha, \\
& V T T_{P T}=\alpha+\pi .
\end{aligned}
$$

\section{Robustness check}

We estimated three additional sets of models: Nested logit models, models with all five alternatives (including 'Bike/Walk' and 'Cancel the trip'), and models with only the two car alternatives. The nested logit models were estimated using the same sample as the one used in section 5. The models with five alternatives include a few additional choices, namely the 53 cases where one of the alternatives 'Bike/Walk' and 'Cancel the trip' was chosen. The models with only the car alternatives were estimated on the subsample of choices where a car alternative was chosen.

The nested logit formulations have one additional parameter compared to the model in section 4: An nest parameter for the nest of car alternatives. The corresponding nest parameter for the PT alternative is normalized to 1. The models with five alternatives have two additional parameters compared to the model in section 4: These are the logarithms of the generalized cost of the alternatives 'Bike/Walk' and 'Cancel the trip', which are assumed to be constant over respondents and choices. The models with only car alternatives include only a subset of the parameters of the model in sections 4 , as the PT-parameters $K, \lambda$ and $\pi$ are not estimated.

Tables 8 - 16 provide the results of the three sets of models. 
Table 8: Estimation Summary: Nested logit models, with multiplicative error terms

\begin{tabular}{|c|c|c|c|c|c|c|}
\hline \multirow[b]{2}{*}{ Sample } & \multirow[b]{2}{*}{ Specification } & \multirow[b]{2}{*}{$\mathrm{N}$ par } & \multirow[b]{2}{*}{$\mathrm{N}$ obs } & \multicolumn{3}{|c|}{ Multiplicative errors } \\
\hline & & & & LL & AIC & $\mathrm{BIC}$ \\
\hline All & exp-exp & 9 & 1547 & -1282.81 & 2583.61 & 2631.71 \\
\hline All & const-exp & 8 & 1547 & -1286.51 & 2589.03 & 2631.78 \\
\hline All & const-affine & 7 & 1547 & -1286.79 & 2587.57 & 2624.98 \\
\hline All & const-step & 8 & 1547 & -1283.47 & 2582.94 & 2625.69 \\
\hline Fixed work time & exp-exp & 9 & 528 & -424.95 & 867.89 & 906.31 \\
\hline Fixed work time & const-exp & 8 & 528 & -426.63 & 869.25 & 903.41 \\
\hline Fixed work time & const-affine & 7 & 528 & -426.74 & 867.48 & 897.37 \\
\hline Fixed work time & const-step & 8 & 528 & -422.14 & 860.27 & 894.42 \\
\hline Flex work time & exp-exp & 9 & 1019 & -825.20 & 1668.39 & 1712.73 \\
\hline Flex work time & const-exp & 8 & 1019 & -828.11 & 1672.22 & 1711.63 \\
\hline Flex work time & const-affine & 7 & 1019 & -828.32 & 1670.65 & 1705.13 \\
\hline Flex work time & const-step & 8 & 1019 & -830.23 & 1676.46 & 1715.87 \\
\hline
\end{tabular}

LL: Log likelihood value at convergence

AIC: Akaike information criterion

BIC: Bayesian information criterion 
Table 9: Parameter estimates with robust standard errors (nested logit models with multiplicative error terms).

\begin{tabular}{|c|c|c|c|c|c|c|c|c|c|}
\hline Specification & \multirow{2}{*}{\multicolumn{3}{|c|}{$\begin{array}{c}\text { Exp-Exp } \\
\text { All }\end{array}$}} & \multicolumn{3}{|c|}{ Exp-Exp } & \multicolumn{3}{|c|}{ Exp-Exp } \\
\hline Sample & & & & \multicolumn{3}{|c|}{ Fixed work time } & \multicolumn{3}{|c|}{ Flex work time } \\
\hline Parameter & Estimate & Std Err & & Estimate & Std Err & & Estimate & Std Err & \\
\hline$\mu$ (error scale) & 2.41 & 0.29 & $* * *$ & 1.44 & 0.428 & $* * *$ & 3.28 & 0.402 & $* * *$ \\
\hline$\mu_{\text {car }}$ (nest scale) & 1.23 & 0.215 & $* * *$ & 0.92 & 0.233 & $* * *$ & 1.13 & 0.202 & $* * *$ \\
\hline$n$ & -5.23 & 12.8 & & -2.24 & 1.64 & & -10.2 & 37.3 & \\
\hline$m$ & 9.10 & 23.1 & & 5.53 & 6.72 & & 9.29 & 34.4 & \\
\hline$\eta \cdot 1000$ & 1.82 & 4.59 & & 10.5 & 12.2 & & 0.911 & 3.35 & \\
\hline$W_{0}$ & -8.06 & 23.1 & & -5.68 & 6.42 & & -8.02 & 34.4 & \\
\hline$K$ & 36.3 & 7.86 & $* * *$ & 15.2 & 10.6 & & 47.0 & 8.16 & $* * *$ \\
\hline$\lambda$ & 1.87 & 0.414 & $* * *$ & 2.21 & 1.06 & $* *$ & 1.41 & 0.304 & $* * *$ \\
\hline$\pi$ & -0.458 & 0.183 & $* *$ & 0.204 & 0.29 & & -0.683 & 0.182 & $* * *$ \\
\hline Specification & \multicolumn{3}{|c|}{ Const-Exp } & \multicolumn{3}{|c|}{ Const-Exp } & \multicolumn{3}{|c|}{ Const-Exp } \\
\hline Sample & \multicolumn{3}{|c|}{ All } & \multicolumn{3}{|c|}{ Fixed work time } & \multicolumn{3}{|c|}{ Flex work time } \\
\hline Parameter & Estimate & Std Err & & Estimate & Std Err & & Estimate & Std Err & \\
\hline$\mu$ (error scale) & 2.41 & 0.299 & $* * *$ & 1.56 & 0.398 & $* * *$ & 3.30 & 0.419 & $* * *$ \\
\hline$\mu_{\text {car }}$ (nest scale) & 1.16 & 0.202 & $* * *$ & 0.999 & 0.258 & $* * *$ & 1.04 & 0.193 & $* * *$ \\
\hline$m$ & 6.18 & 7.72 & & 16.1 & 33.9 & & 3.27 & 4.9 & \\
\hline$\eta \cdot 1000$ & 3.93 & 4.95 & & 4.37 & 9.58 & & 4.76 & 7.12 & \\
\hline$W_{0}$ & -5.02 & 7.69 & & -15.7 & 33.8 & & -1.91 & 4.87 & \\
\hline$K$ & 32.5 & 7.41 & $* * *$ & 6.75 & 9.33 & & 43.4 & 7.62 & $* * *$ \\
\hline$\lambda$ & 1.86 & 0.425 & $* * *$ & 2.27 & 1.05 & $* *$ & 1.37 & 0.307 & $* * *$ \\
\hline$\pi$ & -0.337 & 0.165 & $* *$ & 0.442 & 0.279 & & -0.579 & 0.163 & $* * *$ \\
\hline
\end{tabular}

*** denotes significance at the $1 \%$ level, $* *$ at the $5 \%$ level and $*$ at the $10 \%$ level. 
Table 10: Parameter estimates with robust standard errors (nested logit models with multiplicative error terms).

\begin{tabular}{|c|c|c|c|c|c|c|c|c|c|}
\hline \multirow{4}{*}{$\begin{array}{l}\text { Specification } \\
\text { Sample } \\
\begin{array}{l}\text { Parameter } \\
\mu \text { (error scale) }\end{array}\end{array}$} & \multirow{2}{*}{\multicolumn{3}{|c|}{$\begin{array}{l}\text { Const-Affine } \\
\text { All }\end{array}$}} & \multirow{2}{*}{\multicolumn{3}{|c|}{$\begin{array}{c}\text { Const-Affine } \\
\text { Fixed work time }\end{array}$}} & \multirow{2}{*}{\multicolumn{3}{|c|}{$\begin{array}{l}\text { Const-Affine } \\
\text { Flex work time }\end{array}$}} \\
\hline & & & & & & & & & \\
\hline & \multirow{2}{*}{$\begin{array}{c}\text { Estimate } \\
2.43\end{array}$} & \multicolumn{2}{|l|}{ Std Err } & \multirow{2}{*}{$\begin{array}{c}\text { Estimate } \\
1.62\end{array}$} & \multicolumn{2}{|l|}{ Std Err } & \multirow{2}{*}{$\begin{array}{c}\text { Estimate } \\
3.32\end{array}$} & \multicolumn{2}{|l|}{ Std Err } \\
\hline & & 0.302 & $* * *$ & & 0.359 & $* * *$ & & 0.421 & *** \\
\hline$\mu_{\text {car }}$ (nest scale) & 1.18 & 0.202 & $* * *$ & 1.00 & 0.271 & *** & 1.06 & 0.191 & $* * *$ \\
\hline$\omega$ & 0.0240 & 0.00324 & $* * *$ & 0.0687 & 0.0194 & $* * *$ & 0.0155 & 0.00245 & $* * *$ \\
\hline$\zeta$ & 1.19 & 0.0891 & $* * *$ & 0.424 & 0.25 & $*$ & 1.38 & 0.0938 & *** \\
\hline K & 32.6 & 7.44 & $* * *$ & 7.20 & 9.53 & & 43.5 & 7.65 & $* * *$ \\
\hline$\lambda$ & 1.86 & 0.426 & $* * *$ & 2.17 & 0.926 & $* *$ & 1.37 & 0.307 & *** \\
\hline$\pi$ & -0.343 & 0.166 & $* *$ & 0.411 & 0.27 & & -0.583 & 0.164 & *** \\
\hline Specification & \multicolumn{3}{|c|}{ Const-Step } & \multicolumn{3}{|c|}{ Const-Step } & \multicolumn{3}{|c|}{ Const-Step } \\
\hline Sample & \multicolumn{3}{|c|}{ All } & \multicolumn{3}{|c|}{ Fixed work time } & \multicolumn{3}{|c|}{ Flex work time } \\
\hline Parameter & Estimate & Std Err & & Estimate & Std Err & & Estimate & Std Err & \\
\hline$\mu$ (error scale) & 2.70 & 0.331 & $* * *$ & 1.89 & 0.413 & $* * *$ & 3.54 & 0.451 & *** \\
\hline$\mu_{\text {car }}$ (nest scale) & 3.23 & 0.367 & $* * *$ & 2.48 & 0.510 & $* * *$ & 3.74 & 0.470 & $* * *$ \\
\hline$\alpha$ & 1.05 & 0.0910 & $* * *$ & 0.440 & 0.198 & $* *$ & 1.27 & 0.0956 & *** \\
\hline$\beta$ & 0.533 & 0.0767 & $* * *$ & 1.06 & 0.228 & $* * *$ & 0.363 & 0.0699 & *** \\
\hline$\gamma$ & 0.814 & 0.115 & $* * *$ & 1.70 & 0.352 & $* * *$ & 0.533 & 0.1013 & $* * *$ \\
\hline$K$ & 38.6 & 7.22 & $* * *$ & 15.8 & 12.0 & & 46.7 & 7.32 & $* * *$ \\
\hline$\lambda$ & 1.65 & 0.351 & $* * *$ & 2.16 & 0.861 & $* *$ & 1.27 & 0.275 & $* * *$ \\
\hline$\pi$ & -0.291 & 0.158 & $*$ & 0.501 & 0.318 & & -0.535 & 0.155 & $* * *$ \\
\hline
\end{tabular}

$* * *$ denotes significance at the $1 \%$ level, $* *$ at the $5 \%$ level and * at the $10 \%$ level. 
Table 11: Estimation Summary: Models with all five alternatives, with multiplicative error terms

\begin{tabular}{llrrrrr}
\hline & & & & \multicolumn{3}{c}{ Multiplicative errors } \\
\cline { 5 - 7 } Sample & Specification & N par & N obs & LL & AIC & BIC \\
\hline All & exp-exp & 10 & 1600 & -1546.43 & 3102.85 & 3128.98 \\
All & const-exp & 9 & 1600 & -1548.76 & 3105.52 & 3126.42 \\
All & const-affine & 8 & 1600 & -1549.38 & 3104.75 & 3120.42 \\
All & const-step & 9 & 1600 & -1546.23 & 3100.46 & 3121.36 \\
& & & & & & \\
Fixed work time & exp-exp & 10 & 554 & -541.61 & 1093.21 & 1113.88 \\
Fixed work time & const-exp & 9 & 554 & -543.22 & 1094.45 & 1110.98 \\
Fixed work time & const-affine & 8 & 554 & -543.33 & 1092.66 & 1105.06 \\
Fixed work time & const-step & 9 & 554 & -539.30 & 1086.60 & 1103.14 \\
& & & & & & \\
Flex work time & exp-exp & 10 & 1046 & -969.48 & 1948.96 & 1973.04 \\
Flex work time & const-exp & 9 & 1046 & -971.56 & 1951.12 & 1970.38 \\
Flex work time & const-affine & 8 & 1046 & -971.97 & 1949.93 & 1964.38 \\
Flex work time & const-step & 9 & 1046 & -973.92 & 1955.83 & 1975.10 \\
\hline
\end{tabular}

LL: Log likelihood value at convergence

AIC: Akaike information criterion

BIC: Bayesian information criterion 
Table 12: Parameter estimates with robust standard errors (models with all five alternatives, with multiplicative error terms).

\begin{tabular}{|c|c|c|c|c|c|c|c|c|c|}
\hline \multirow{4}{*}{$\begin{array}{l}\text { Specification } \\
\text { Sample } \\
\text { Parameter } \\
\mu \text { (error scale) }\end{array}$} & \multirow{2}{*}{\multicolumn{3}{|c|}{$\begin{array}{c}\text { Exp-Exp } \\
\text { All }\end{array}$}} & \multirow{2}{*}{\multicolumn{3}{|c|}{$\begin{array}{c}\text { Exp-Exp } \\
\text { Fixed work time }\end{array}$}} & \multirow{2}{*}{\multicolumn{3}{|c|}{$\begin{array}{c}\text { Exp-Exp } \\
\text { Flex work time }\end{array}$}} \\
\hline & & & & & & & & & \\
\hline & \multirow{2}{*}{$\begin{array}{c}\text { Estimate } \\
2.34\end{array}$} & \multicolumn{2}{|l|}{ Std Err } & \multirow{2}{*}{$\begin{array}{c}\text { Estimate } \\
1.36\end{array}$} & \multicolumn{2}{|l|}{ Std Err } & \multirow{2}{*}{$\begin{array}{c}\text { Estimate } \\
3.05\end{array}$} & \multicolumn{2}{|l|}{ Std Err } \\
\hline & & 0.213 & $* * *$ & & 0.412 & $* * *$ & & 0.279 & *** \\
\hline$n$ & -2.05 & 2.44 & & -2.16 & 1.64 & & -4.42 & 16.4 & \\
\hline$m$ & 4.56 & 5.55 & & 5.85 & 7.35 & & 4.38 & 15.7 & \\
\hline$\eta \cdot 1000$ & 4.51 & 5.33 & & 10.0 & 12.1 & & 2.22 & 8.02 & \\
\hline$W_{0}$ & -3.65 & 5.52 & & -5.99 & 7.06 & & -3.17 & 15.7 & \\
\hline K & 35.2 & 7.65 & $* * *$ & 15.0 & 10.8 & & 46.5 & 8.6 & $* * *$ \\
\hline$\lambda$ & 1.81 & 0.276 & $* * *$ & 2.52 & 1.15 & $* *$ & 1.51 & 0.253 & $* * *$ \\
\hline$\pi$ & -0.477 & 0.179 & $* * *$ & 0.201 & 0.287 & & -0.723 & 0.192 & $* * *$ \\
\hline $\ln \mathscr{G}_{\text {bike/walk }}$ & 6.06 & 0.172 & $* * *$ & 6.51 & 0.694 & $* * *$ & 5.94 & 0.175 & $* * *$ \\
\hline $\ln \mathscr{G}_{\text {not travel }}$ & 5.62 & 0.126 & $* * *$ & 5.91 & 0.51 & $* * *$ & 5.53 & 0.114 & $* * *$ \\
\hline Specification & \multicolumn{3}{|c|}{ Const-Exp } & \multicolumn{3}{|c|}{ Const-Exp } & \multicolumn{3}{|c|}{ Const-Exp } \\
\hline Sample & \multicolumn{3}{|c|}{ All } & \multicolumn{3}{|c|}{ Fixed work time } & \multicolumn{3}{|c|}{ Flex work time } \\
\hline Parameter & Estimate & Std Err & & Estimate & Std Err & & Estimate & Std Err & \\
\hline$\mu$ (error scale) & 2.34 & 0.213 & $* * *$ & 1.53 & 0.35 & $* * *$ & 2.98 & 0.281 & *** \\
\hline$m$ & 4.63 & 4.02 & & 17.0 & 36.5 & & 2.54 & 2.82 & \\
\hline$\eta \cdot 1000$ & 5.93 & 5.14 & & 4.13 & 9.18 & & 6.72 & 7.31 & \\
\hline$W_{0}$ & -3.57 & 4.00 & & -16.7 & 36.4 & & -1.22 & 2.80 & \\
\hline K & 31.4 & 7.20 & $* * *$ & 7.31 & 9.22 & & 42.4 & 8.12 & $* * *$ \\
\hline$\lambda$ & 1.84 & 0.286 & $* * *$ & 2.32 & 0.852 & $* * *$ & 1.55 & 0.266 & $* * *$ \\
\hline$\pi$ & -0.349 & 0.160 & $* *$ & 0.409 & 0.267 & & -0.587 & 0.175 & *** \\
\hline $\ln \mathscr{G}_{\text {bike/walk }}$ & 6.07 & 0.175 & $* * *$ & 6.33 & 0.502 & $* * *$ & 5.97 & 0.183 & *** \\
\hline $\ln \mathscr{G}_{\text {not travel }}$ & 5.63 & 0.128 & $* * *$ & 5.80 & 0.384 & $* * *$ & 5.55 & 0.118 & $* * *$ \\
\hline
\end{tabular}

$* * *$ denotes significance at the $1 \%$ level, $* *$ at the $5 \%$ level and $*$ at the $10 \%$ level. 
Table 13: Parameter estimates with robust standard errors (models with all five alternatives, with multiplicative error terms).

\begin{tabular}{|c|c|c|c|c|c|c|c|c|c|}
\hline \multirow{4}{*}{$\begin{array}{l}\text { Specification } \\
\text { Sample } \\
\text { Parameter } \\
\mu \text { (error scale) }\end{array}$} & \multirow{2}{*}{\multicolumn{3}{|c|}{$\begin{array}{c}\text { Const-Affine } \\
\text { All }\end{array}$}} & \multirow{2}{*}{\multicolumn{3}{|c|}{$\begin{array}{c}\text { Const-Affine } \\
\text { Fixed work time }\end{array}$}} & \multirow{2}{*}{\multicolumn{3}{|c|}{$\begin{array}{l}\text { Const-Affine } \\
\text { Flex work time }\end{array}$}} \\
\hline & & & & & & & & & \\
\hline & \multirow{2}{*}{$\begin{array}{c}\text { Estimate } \\
2.39\end{array}$} & \multicolumn{2}{|l|}{ Std Err } & \multirow{2}{*}{$\begin{array}{c}\text { Estimate } \\
1.59\end{array}$} & \multicolumn{2}{|l|}{ Std Err } & \multirow{2}{*}{$\begin{array}{c}\text { Estimate } \\
3.03\end{array}$} & \multicolumn{2}{|l|}{ Std Err } \\
\hline & & 0.205 & $* * *$ & & 0.312 & $* * *$ & & 0.273 & $* * *$ \\
\hline$\omega$ & 0.0272 & 0.00365 & $* * *$ & 0.0690 & 0.0170 & *** & 0.0170 & 0.00273 & $* * *$ \\
\hline$\zeta$ & 1.10 & 0.0887 & $* * *$ & 0.379 & 0.184 & $* *$ & 1.34 & 0.0999 & *** \\
\hline K & 31.8 & 7.16 & $* * *$ & 7.74 & 9.38 & & 42.6 & 8.08 & $* * *$ \\
\hline$\lambda$ & 1.80 & 0.271 & $* * *$ & 2.23 & 0.725 & *** & 1.53 & 0.257 & $* * *$ \\
\hline$\pi$ & -0.367 & 0.158 & $* *$ & 0.382 & 0.260 & & -0.599 & 0.173 & $* * *$ \\
\hline $\ln \mathscr{G}_{\text {bike/walk }}$ & 6.04 & 0.167 & $* * *$ & 6.27 & 0.434 & *** & 5.96 & 0.178 & $* * *$ \\
\hline $\ln \mathscr{G}_{\text {not travel }}$ & 5.62 & 0.123 & $* * *$ & 5.76 & 0.337 & *** & 5.54 & 0.115 & *** \\
\hline Specification & \multicolumn{3}{|c|}{ Const-Step } & \multicolumn{3}{|c|}{ Const-Step } & \multicolumn{3}{|c|}{ Const-Step } \\
\hline Sample & \multicolumn{3}{|c|}{ All } & \multicolumn{3}{|c|}{ Fixed work time } & \multicolumn{3}{|c|}{ Flex work time } \\
\hline Parameter & Estimate & Std Err & & Estimate & Std Err & & Estimate & Std Err & \\
\hline$\mu$ (error scale) & 2.68 & 0.222 & $* * *$ & 2.14 & 0.329 & *** & 3.23 & 0.290 & $* * *$ \\
\hline$\alpha$ & 0.950 & 0.0888 & $* * *$ & 0.332 & 0.162 & $* *$ & 1.22 & 0.0992 & $* * *$ \\
\hline$\beta$ & 0.586 & 0.0868 & $* * *$ & 1.17 & 0.237 & *** & 0.384 & 0.0779 & $* * *$ \\
\hline$\gamma$ & 0.921 & 0.122 & $* * *$ & 1.72 & 0.323 & $* * *$ & 0.592 & 0.109 & $* * *$ \\
\hline$K$ & 38.2 & 6.99 & $* * *$ & 19.5 & 11.0 & $*$ & 45.9 & 7.78 & $* * *$ \\
\hline$\lambda$ & 1.59 & 0.222 & $* * *$ & 1.67 & 0.411 & $* * *$ & 1.41 & 0.230 & $* * *$ \\
\hline$\pi$ & -0.303 & 0.150 & $* *$ & 0.377 & 0.263 & & -0.540 & 0.165 & *** \\
\hline $\ln \mathscr{C}_{\text {bike/walk }}$ & 5.88 & 0.144 & $* * *$ & 5.84 & 0.270 & *** & 5.86 & 0.165 & $* * *$ \\
\hline $\ln \mathscr{G}_{\text {not travel }}$ & 5.50 & 0.106 & $* * *$ & 5.46 & 0.215 & *** & 5.47 & 0.106 & $* * *$ \\
\hline
\end{tabular}

*** denotes significance at the $1 \%$ level, $* *$ at the $5 \%$ level and $*$ at the $10 \%$ level. 
Table 14: Estimation Summary: Models with car alternatives only, with multiplicative error terms

\begin{tabular}{lllllrr}
\hline & & & & \multicolumn{3}{c}{ Multiplicative errors } \\
\cline { 5 - 7 } Sample & Specification & N par & N obs & LL & AIC & BIC \\
\hline All & exp-exp & 5 & 1373 & no conv & - & - \\
All & const-exp & 4 & 1373 & -856.40 & 1720.80 & 1741.70 \\
All & const-affine & 3 & 1373 & -856.46 & 1718.92 & 1734.59 \\
All & const-step & 4 & 1373 & -857.82 & 1723.64 & 1744.54 \\
& & & & & & \\
Fixed work time & exp-exp & 5 & 461 & -259.31 & 528.63 & 549.29 \\
Fixed work time & const-exp & 4 & 461 & -260.51 & 529.02 & 545.56 \\
Fixed work time & const-affine & 3 & 461 & -260.63 & 527.26 & 539.66 \\
Fixed work time & const-step & 4 & 461 & -258.13 & 524.26 & 540.80 \\
& & & & & & \\
Flex work time & exp-exp & 5 & 912 & no conv & - & - \\
Flex work time & const-exp & 4 & 912 & -575.52 & 1159.04 & 1178.30 \\
Flex work time & const-affine & 3 & 912 & -575.55 & 1157.09 & 1171.54 \\
Flex work time & const-step & 4 & 912 & -579.04 & 1166.07 & 1185.33 \\
\hline
\end{tabular}

LL: Log likelihood value at convergence

AIC: Akaike information criterion

BIC: Bayesian information criterion 
Table 15: Parameter estimates with robust standard errors (models with car alternatives only, with multiplicative error terms).

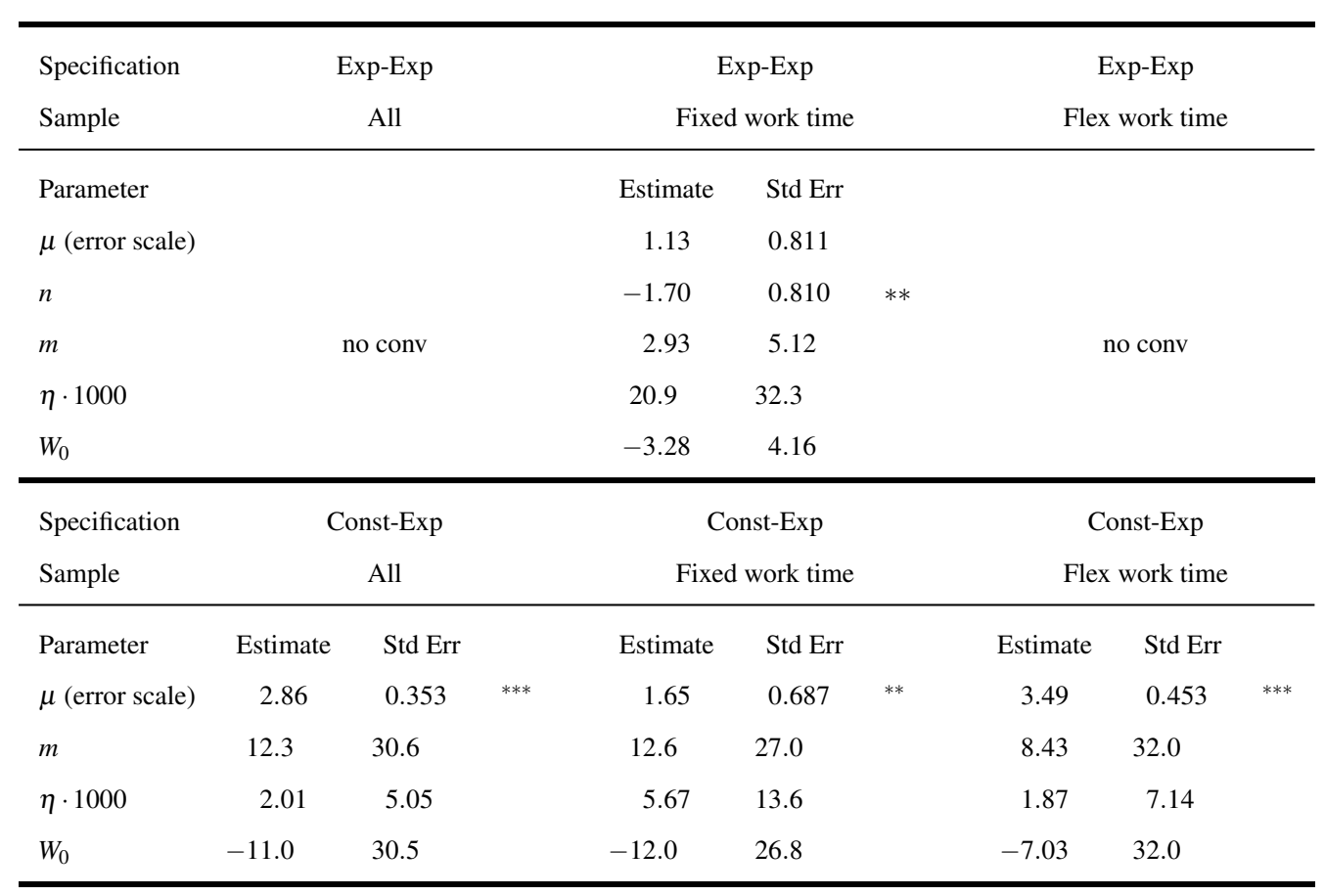

*** denotes significance at the $1 \%$ level, $* *$ at the $5 \%$ level and $*$ at the $10 \%$ level. 
Table 16: Parameter estimates with robust standard errors (models with car alternatives only, with multiplicative error terms).

\begin{tabular}{|c|c|c|c|c|c|c|c|c|c|}
\hline Specification & \multirow{2}{*}{\multicolumn{3}{|c|}{$\begin{array}{c}\text { Const-Affine } \\
\text { All }\end{array}$}} & \multicolumn{3}{|c|}{ Const-Affine } & \multicolumn{3}{|c|}{ Const-Affine } \\
\hline Sample & & & & \multicolumn{3}{|c|}{ Fixed work time } & \multicolumn{3}{|c|}{ Flex work time } \\
\hline Parameter & Estimate & Std Err & & Estimate & Std Err & & Estimate & Std Err & \\
\hline$\mu$ (error scale) & 2.89 & 0.344 & $* * *$ & 1.82 & 0.529 & $* * *$ & 3.51 & 0.445 & $* * *$ \\
\hline$\omega$ & 0.0244 & 0.00345 & $* * *$ & 0.0661 & 0.0209 & $* * *$ & 0.0157 & 0.00259 & $* * *$ \\
\hline$\zeta$ & 1.24 & 0.0882 & $* * *$ & 0.606 & 0.244 & $* *$ & 1.4 & 0.0962 & $* * *$ \\
\hline Specification & \multicolumn{3}{|c|}{ Const-Step } & \multicolumn{3}{|c|}{ Const-Step } & \multicolumn{3}{|c|}{ Const-Step } \\
\hline Sample & \multicolumn{3}{|c|}{ All } & \multicolumn{3}{|c|}{ Fixed work time } & \multicolumn{3}{|c|}{ Flex work time } \\
\hline Parameter & Estimate & Std Err & & Estimate & Std Err & & Estimate & Std Err & \\
\hline$\mu$ (error scale) & 3.35 & 0.366 & $* * *$ & 2.64 & 0.584 & $* * *$ & 3.81 & 0.468 & $* * *$ \\
\hline$\alpha$ & 1.12 & 0.0918 & $* * *$ & 0.542 & 0.226 & $* *$ & 1.31 & 0.101 & $* * *$ \\
\hline$\beta$ & 0.550 & 0.0807 & $* * *$ & 1.03 & 0.229 & $* * *$ & 0.381 & 0.0747 & $* * *$ \\
\hline$\gamma$ & 0.762 & 0.120 & $* * *$ & 1.70 & 0.447 & $* * *$ & 0.492 & 0.107 & $* * *$ \\
\hline
\end{tabular}

$* * *$ denotes significance at the $1 \%$ level, $* *$ at the $5 \%$ level and $*$ at the $10 \%$ level. 